OPEN ACCESS

Edited by:

Rustam Aminov,

University of Aberdeen,

United Kingdom

Reviewed by:

George Grant,

University of Aberdeen,

United Kingdom

Ryo Inoue,

Setsunan University, Japan

Dale Nicholas Gerding,

Edward Hines, Jr. VA Hospital,

United States

*Correspondence:

Rossen Donev

rossen.donev@micropharm.co.uk

tThese authors have contributed equally to this work

Specialty section:

This article was submitted to

Infectious Diseases,

a section of the journal

Frontiers in Microbiology

Received: 01 July 2020

Accepted: 31 August 2020

Published: 22 September 2020

Citation:

Roberts AK, Harris HC, Smith M, Giles J, Polak O, Buckley AM, Clark E,

Ewin D, Moura IB, Spitall W,

Shone CC, Wilcox M, Chilton $C$ and

Donev R (2020) A Novel, Orally

Delivered Antibody Therapy and lts

Potential to Prevent Clostridioides

difficile Infection in Pre-clinical

Models. Front. Microbiol. 11:578903.

doi: 10.3389/fmicb.2020.578903

\section{A Novel, Orally Delivered Antibody Therapy and Its Potential to Prevent Clostridioides difficile Infection in Pre-clinical Models}

\author{
April K. Roberts ${ }^{1 \dagger}$, Hannah C. Harris ${ }^{2 \dagger}$, Michael Smith ${ }^{1}$, Joanna Giles ${ }^{3}$, Oktawia Polak ${ }^{3}$, \\ Anthony M. Buckley'2, Emma Clark'2, Duncan Ewin'², Ines B. Moura ${ }^{2}$, William Spitall2, \\ Clifford C. Shone ${ }^{1}$, Mark Wilcox $^{2,4}$, Caroline Chilton ${ }^{2}$ and Rossen Donev ${ }^{3 *}$ \\ 1 Toxins Group, National Infection Service, Public Health England, Porton Down, United Kingdom, ${ }^{2}$ Leeds Institute \\ of Medical Research, Faculty of Medicine and Health, University of Leeds, Leeds, United Kingdom, ${ }^{3}$ MicroPharm Ltd., \\ Newcastle Emlyn, United Kingdom, ${ }^{4}$ Department of Microbiology, Leeds Teaching Hospitals NHS Trust, Leeds General \\ Infirmary, Leeds, United Kingdom
}

Clostridioides difficile infection (CDI) is a toxin-mediated infection in the gut and a major burden on healthcare facilities worldwide. We rationalized that it would be beneficial to design an antibody therapy that is delivered to, and is active at the site of toxin production, rather than neutralizing the circulating and luminal toxins after significant damage of the layers of the intestines has occurred. Here we describe a highly potent therapeutic, OraCAb, with high antibody titers and a formulation that protects the antibodies from digestion/inactivation in the gastrointestinal tract. The potential of OraCAb to prevent $\mathrm{CDI}$ in an in vivo hamster model and an in vitro human colon model was assessed. In the hamster model we optimized the ratio of the antibodies against each of the toxins produced by $\mathrm{C}$. difficile (Toxins $\mathrm{A}$ and $\mathrm{B}$ ). The concentration of immunoglobulins that is effective in a hamster model of CDI was determined. A highly significant difference in animal survival for those given an optimized OraCAb formulation versus an untreated control group was observed. This is the first study testing the effect of oral antibodies for treatment of CDI in an in vitro gut model seeded with a human fecal inoculum. Treatment with OraCAb successfully neutralized toxin production and did not interfere with the colonic microbiota in this model. Also, treatment with a combination of vancomycin and OraCAb prevented simulated CDI recurrence, unlike vancomycin therapy alone. These data demonstrate the efficacy of OraCAb formulation for the treatment of CDI in pre-clinical models.

Keywords: Clostridioides difficile infection (CDI), immunotherapy of CDI, oral antibodies, formulation protecting antibodies from digestion/inactivation, in vivo hamster model of $\mathrm{CDI}$, in vitro human gut model of CDI

\section{INTRODUCTION}

Clostridioides difficile infection (CDI) is a leading cause of infectious antibiotic associated diarrhea and a major burden on healthcare facilities worldwide (Wiegand et al., 2012; Lessa et al., 2015; Guh et al., 2020). Disruption to the normal intestinal microbiota (e.g., by antibiotic exposure) is a key risk factor for CDI, leading to loss of colonization resistance and creating a niche which $C$. difficile 
can exploit. CDI is a toxin mediated disease, with the primary disease-causing factors identified as two large protein toxins, Toxin A (TcdA) and Toxin B (TcdB) (Lyerly et al., 1985). A third toxin which is likely to contribute to the disease (Gerding et al., 2014), binary toxin (CDT), has been identified in a number of epidemic strains, however, CDT itself does not appear to be sufficient to cause CDI in animal models (Kuehne et al., 2014).

Currently, the standard treatment for CDI is antibiotic therapy (use of metronidazole, vancomycin and more recently, fidaxomicin), which has limited long-term efficacy, with approximately $20-30 \%$ of patients experiencing recurrent disease (Kelly, 2012) and fidaxomicin therapy resulting in $\sim 12 \%$ less recurrences compared to vancomycin treatment (Mullane, 2014). Alternative therapies directed at neutralizing TcdA and TcdB and restoring the disrupted microbiome are in development. These include antibody-based therapies, vaccines and fecal microbiome transfer (FMT) and are summarized by Madoff et al. (2019). Bezlotoxumab developed by Merck is the first monoclonal antibody approved to prevent the recurrence of $C$. difficile infection (Lee et al., 2017). It should be noted that despite the promising benefits reported for FMT, the transmission of an extended-spectrum beta-lactamase producing Escherichia coli to two patients in two independent clinical trials has been reported. Both patients developed bacteraemia and one died. Therefore, the screening of FMT donors requires improvement (DeFilipp et al., 2019).

It is likely that the host immune response is a critical factor in recurrent disease, with naturally occurring toxin antibodies shown to be protective against recurrent CDI (Kyne et al., 2001). Human monoclonal antibodies (MAbs) against TcdA and TcdB (actoxumab and bezlotoxumab) have been developed (Babcock et al., 2006) and systemic administration of these antibodies alongside standard of care therapy has been tested in two large international multicentre, double blind randomized placebo controlled trials (Wilcox et al., 2017). Administration of bezlotoxumab led to significantly lower rates $(\sim 11 \%$ less as an absolute value compared to placebo) of recurrent disease 12 weeks after administration. Administration of actoxumab alone showed no clinical benefit, and there was no additional advantage of a combination of actoxumab and bezlotoxumab compared with bezlotoxumab alone (Wilcox et al., 2017). Thus, the therapeutic use of some MAbs against $C$. difficile toxins has been shown to be beneficial in reducing cases of recurrent disease when administered systemically. This MAb-based therapy has been licensed for the prevention of recurrent CDI (when used with standard of care antibiotics) and marketed as Zinplava.

Previously, we have described the use of systemically delivered ovine polyclonal antibodies raised against toxoids of TcdA and $\mathrm{TcdB}$ and against non-toxic recombinant fragments of TcdA (TxA4) and TcdB (TxB4) as a potential treatment for severe CDI. Importantly, these polyclonal antibodies can neutralize toxins from several circulating $C$. difficile toxinotypes ( 0 , III, V) (Roberts et al., 2012; Maynard-Smith et al., 2014). In the present study, we describe the potential of these antibodies for the management of CDI when delivered as an oral therapy. The antibodies could be used alongside standard of care antibiotics and in preference to or in combination with FMT for the treatment of recurrence. This oral therapy also has the potential to be used as a prophylactic in high risk patients when entering a healthcare facility.

For toxin-mediated infections in the gut such as CDI, it would be preferable to design an antibody therapy that is delivered to and is active at the site of toxin production. The rationale for delivering the antibodies orally is to target directly the site of infection where $\mathrm{TcdA}$ and $\mathrm{TcdB}$ are released, rather than neutralizing the toxins in the circulation and gut lamina propria after significant damage of layers of the intestines has occurred. Furthermore, especially when mucosal inflammation is absent, only a negligible proportion of immunoglobulins injected systemically may reach the lumen of the gut by transudation from serum (Shearman et al., 1972). The development of several orally delivered antibody-based therapies for CDI have been described previously. These therapies include toxin-neutralizing antibodies, for example, chicken IgY (Kink and Williams, 1998) and IgG in bovine colostrum (Sponseller et al., 2015; Hutton et al., 2017).

Using platform technologies established by MicroPharm and Public Health England (PHE), we have developed OraCAb, a novel orally delivered ovine polyclonal antibody product targeted toward $C$. difficile toxins. OraCAb has several advantages over a therapy based on bovine colostrum. For example, there is a constant supply of hyperimmune serum from immunized sheep from which to prepare the product. In addition, OraCAb contains highly efficacious toxin-neutralizing antibodies purified from serum. Furthermore, bovine colostrum derived antibodies, currently used for trials, are not protected against denaturation and digestion by acidic gastric juice and pepsin in the stomach, and trypsin and chymotrypsin in the small intestine. OraCAb has been formulated to maintain the integrity of the antibodies whilst the therapeutic is passing through the stomach and small intestine. Unlike in intravenous administration, polyclonal antibodies are not expected to cause a significant systemic humoral immune response when taken orally. A recently published study (Crowe et al., 2018) demonstrated that a small amount of orally administered antibodies gain access to the lamina propria and serum only when intestinal lesions are in place, with antibody concentrations increasing with disease severity. OraCAb is intended for treatment of mild to moderate CDI where severe lesions are unlikely to occur. Neutralization of TcdA and TcdB at the site of their production will eliminate further damage in the colon and should minimize infiltration of orally administered polyclonal immunoglobulins. The major advantages of using polyclonal rather than MAbs against TcdA and $\mathrm{TcdB}$ are the greater avidity of polyclonal antibodies, more efficient neutralization of the very large toxin molecules and their ability to neutralize a variety of toxinotypes of TcdA and TcdB.

This work describes the development strategy used to formulate OraCAb and its potential to prevent CDI and CDI recurrence in pre-clinical in vitro and in vivo models.

\section{MATERIALS AND METHODS}

\section{Study Design}

This study was designed to examine the protective efficacy of an ovine antibody-based, orally delivered therapeutic for the 
treatment of CDI, both in vitro and in the Golden Syrian hamster model. The antibody formulation, OraCAb, was developed to protect the antibodies during transit through the gut and to be suitable for oral administration to hamsters. The formulations were thoroughly tested in vitro before testing in vivo. The in vivo model was employed to demonstrate the efficacy of a variety of OraCAb formulations. The animals used in these studies were Golden Syrian hamsters obtained from a UK Home Office approved breeder (Envigo). The animals were 7-9 weeks old at the start of an experiment, weighing 80-100 g.

The Project Licenses (30-3042 and P66F2D25B) enabling these studies were approved by the Ethical Review Process of Public Health England (PHE), Porton, Salisbury, United Kingdom and the Home Office, United Kingdom. Animals were housed as single sex pairs according to the Home Office Code of Practice for the Housing and Care of Animals Bred, Supplied or Used for Scientific Purposes, December 2014 and the NC3Rs (National Centre for the Replacement, Refinement and Reduction of Animals in Research).

The collection and use of stool samples from healthy adult individuals in the in vitro human gut model was approved by the University of Leeds School of Medicine Research Ethics Committee (Ref: MREC15-070).

\section{Production of Ovine Anti-TxA4 and Anti-TxB4 Antisera and Antibodies}

The recombinant antigens, TxA4 and TxB4 with sequences obtained from VPI10463 strain, were expressed and purified as described by Maynard-Smith et al. (2014). For antiserum production, sheep were immunized at $0,4,8,12$, and 16 weeks with $250 \mu \mathrm{g}$ of either antigen per dose as described in Roberts et al. (2012). Pre-bleeds were collected at week 0 and test bleeds collected at 6, 10, and 14 weeks. A larger bleed was collected at 18 weeks when antibody toxin neutralization titers were high. Prior to immunization, the antigens were treated with formaldehyde, as previously described (Maynard-Smith et al., 2014).

\section{Purification of Bowman-Birk Protease Inhibitor (BBI) From Lima Beans}

$\mathrm{BBI}$ was produced at PHE from commercially available Lima beans. Briefly, Lima beans were blended to a flour and defatted with ethanol. BBI was purified through a combination of aqueous acid extraction, ammonium sulfate precipitation, differential solubilization in ethanol solutions, immobilized metal affinity and anion exchange chromatography. Approximately $2.6 \mathrm{mg}$ of inhibitor, $>95 \%$ pure, was obtained per gram of Lima beans. The inhibitor was dialyzed against $\mathrm{pH} 7.5$ HEPES $(10 \mathrm{mM})$, $\mathrm{NaCl}(100 \mathrm{mM})$, sterile filtered and freeze dried for inclusion in OraCAb formulations, as required. Additional information on BBI purification is available in Supplementary Materials.

\section{Preparation of OraCAb Formulations}

Series of OraCAb formulations used to optimize the anti-TxA4: anti-TxB4 IgG ratio, IgG concentration and comparison of protease inhibitors are given in Table 1.
TABLE 1 | OraCAb formulations used in in vivo studies.

\begin{tabular}{|c|c|c|c|}
\hline \multirow[t]{2}{*}{ Study } & \multicolumn{3}{|c|}{ OraCAb for in vivo studies (concentration } \\
\hline & $\begin{array}{c}\text { Anti-TxB4 } \\
\text { IgG }\end{array}$ & $\begin{array}{c}\text { Anti-TxA4 } \\
\text { IgG }\end{array}$ & $\begin{array}{r}\text { Protease Inhibitor (d } \\
\text { white [DEW] or Bowr } \\
\text { inhibitor [BB }\end{array}$ \\
\hline \multicolumn{4}{|l|}{ A (in vivo) } \\
\hline $\operatorname{DEW}(1: 1 \mathrm{~B}: \mathrm{A})$ & 75 & 75 & DEW 60 \\
\hline DEW (3:1 B:A) & 112.5 & 37.5 & DEW 60 \\
\hline $\mathrm{BBI}(3: 1 \mathrm{~B}: \mathrm{A})$ & 112.5 & 37.5 & $\mathrm{BBI} 10$ \\
\hline \multicolumn{4}{|l|}{ B/D (in vivo) } \\
\hline No DEW 150 mg/ml & 112.5 & 37.5 & DEW 0 \\
\hline $150 \mathrm{mg} / \mathrm{ml}$ & 112.5 & 37.5 & DEW 60 \\
\hline 100 mg/ml & 75 & 25 & DEW 60 \\
\hline $50 \mathrm{mg} / \mathrm{ml}$ & 37.5 & 12.5 & DEW 60 \\
\hline \multicolumn{4}{|l|}{ C (in vivo) } \\
\hline B only & 112.5 & 0 & DEW 60 \\
\hline $3: 1 \mathrm{~B}: \mathrm{A}$ & 112.5 & 37.5 & DEW 60 \\
\hline 1:1 B:A & 75 & 75 & DEW 60 \\
\hline
\end{tabular}

Additional ingredients

Magnesium hydroxide $(23.4 \mathrm{mg} / \mathrm{ml})$, Dried aluminum hydroxide $(26.4 \mathrm{mg} / \mathrm{ml})$, Methylparaben (E218) (2 mg/ml), Propylparaben (E216) $(0.6 \mathrm{mg} / \mathrm{ml})$, Glycine $(15 \mathrm{mg} / \mathrm{ml})$, Simethicone $(16.9 \mathrm{mg} / \mathrm{ml})$, Sodium saccharin $(0.4 \mathrm{mg} / \mathrm{ml})$, and Mannitol $(21 \mathrm{mg} / \mathrm{ml})$.

Anti-TxA4 IgG and anti-TxB4 IgG were formulated in $20 \mathrm{mM}$ sodium citrate buffer ( $\mathrm{pH}$ 6.0) containing $153 \mathrm{mM} \mathrm{NaCl}$ (SCS). After mixing the two antibodies in the required ratios (as described in Table 1), all other constituents of the OraCAb formulation were added and a stable suspension was prepared by mixing with a ONE-ST-C laboratory scale propeller mixer (Joshua Greaves \& Sons Ltd, United Kingdom). The final volume was adjusted with SCS. For the in vitro gut model studies, antibodies formulated in SCS buffer only were used.

\section{$N \alpha$-Benzoyl-DL-Arginine-p-Nitroanilide (BAPNA) Assay for Activity of Trypsin Inhibitors}

The activity of the BBI and dried egg white (DEW) trypsin inhibitors was determined by analysis of the reaction with BAPNA as a substrate for trypsin. Samples of the BBI and DEW were diluted in twofold series in a 96-well plate in quadruplet rows. One hundred $\mu \mathrm{l}$ of porcine trypsin (Sigma) dissolved in $1 \mathrm{mM} \mathrm{HCl}$ (Merck) at concentration of $0.0125 \mathrm{mg} / \mathrm{ml}$ was added to triplicate samples. As a negative control $1 \mathrm{mM} \mathrm{HCl}$ was added to one row of samples. Reagents in the experimental plates were mixed by shaking for $15 \mathrm{~min}$ at room temperature. One hundred $\mu \mathrm{l}$ of $6 \mathrm{mM}$ BAPNA (Sigma) was added to each well and incubated for $30 \mathrm{~min}$ at $37^{\circ} \mathrm{C}$. Fifty $\mu \mathrm{l}$ of $30 \%$ acetic acid was added to each well and the absorbance was read at 410 and $690 \mathrm{~nm}$ using a POLARstar microplate reader (BMG Labtech). Data were normalized by calculating A410-A690 in Microsoft Excel for each well, and by subtracting the values for corresponding samples without trypsin (1 $\mathrm{mM} \mathrm{HCl}$ control). Curves were plotted in Excel and EC $_{50}$ values were generated using a linear regression of data from the assay linear range. 


\section{Stability of Immunoglobulins in OraCAb to Simulated Gastric Fluid (SGF)}

Simulated gastric fluid, $\mathrm{pH} 1.2$ was prepared according to the US Pharmacopeia and contained $2 \mathrm{~g} \mathrm{NaCl}, 7 \mathrm{ml} \mathrm{37 \%} \mathrm{HCl}$ and $3.2 \mathrm{~g}$ porcine pepsin (8000 U) (Sigma) made up to a liter with water for irrigation. OraCAb was evaluated for stability of immunoglobulins to SGF. SGF was mixed with OraCAb formulation in different ratios (10\% OraCAb, 20\% OraCAb, $30 \%$ OraCAb, $40 \%$ OraCAb, and 50\% OraCAb in SGF). The mixtures were incubated for $2.5 \mathrm{~h}$ at $37^{\circ} \mathrm{C}$ with gentle shaking every $15 \mathrm{~min}$. At the end of the incubation, $\mathrm{pH}$ was increased by adding Phosphate Buffered Saline (PBS) to prevent further protein digestion by the pepsin in SGF. Samples were then analyzed by the toxin neutralization assay.

\section{Toxin Neutralization Assay}

Toxin neutralization assays were performed as described previously (Roberts et al., 2012), with modification. Vero cells were seeded at $9 \times 10^{3}$ cells per well in 96-well black microtiterplates (flat bottom, VWR) in Dulbecco's Modified Eagle's Medium (DMEM) supplemented with Penicillin-Streptomycin (100 U/ml and $0.1 \mathrm{mg} / \mathrm{ml}$, respectively; Sigma), Glutamine (2.0 mM; Sigma), HEPES (25 mM Sigma) and 10\% Fetal Bovine Serum (Sigma), and incubated for $24 \mathrm{~h}$ at $37^{\circ} \mathrm{C}$ in a humidified incubator under $5 \% \mathrm{CO}$. Purified TcdA or TcdB proteins were diluted in DMEM and prepared as $40 \mathrm{ng} / \mathrm{ml}$ or $400 \mathrm{pg} / \mathrm{ml}$ dilutions, respectively. Suitably diluted oral formulation, antiTxA4 and anti-TxB4 antibodies were pre-mixed with either TcdA or TcdB in a 96-well culture plate and $100 \mu \mathrm{l}$ of the pre-mix was transferred to the corresponding wells of the prepared plates with Vero cells. Plates were incubated at $37^{\circ} \mathrm{C}$ in $5 \% \mathrm{CO} 2$ for $48 \mathrm{~h}$. Each dilution was performed in duplicate. Forty $\mu \mathrm{l}$ of CellTiter blue stain (Promega) was added to each well and incubated for a further $4 \mathrm{~h}$ to determine cell viability. Fluorescence was determined using a POLARstar microplate reader (BMG Labtech) at $\lambda 590 \mathrm{~nm}$. The cell survival percentage was calculated in Microsoft Excel and plotted for each concentration using GraphPad Prism. EC 50 values were calculated using non-linear regression (Sigmoidal fit, 4PL).

\section{Bacterial Strains}

Two C. difficile strains were used in this study.

The highly virulent VPI 10463 strain (ribotype 087/toxinotype 0 ) was obtained from the American Type Culture Collection (ATCC 43255). Spores were prepared from this strain and used in in vivo models as previously described (Roberts et al., 2012). The VPI10463 strain is a high producer of both TcdA and TcdB that makes the hamster model of CDI described below a very stringent test for the efficacy of OraCAb.

The epidemic 027210 strain (BI/NAP1/PCR ribotype 027/toxinotype III) was originally isolated during an outbreak of $\mathrm{CDI}$ at the Maine Medical Centre (Portland, ME, United States) in 2005 and was kindly supplied by Dr Robert Owens. Spores were prepared from this strain and used in in vitro gut models as previously described (Freeman et al., 2003). The strain used in the in vitro studies was chosen as it is an example of an epidemic, so-called 'hypervirulent' ribotype 027. Importantly, we have a wealth of previous gut model data using this strain, so we can make meaningful comparisons with previous data. This is particularly helpful with a system such as this gut model which is time consuming and labor intensive, so multiple experimental repeats are not feasible.

\section{Animal Model for C. difficile Infection}

The Syrian hamster model was used as described previously (Roberts et al., 2012; Maynard-Smith et al., 2014), using groups of 10 animals (Figure 1). All animals received clindamycin, orogastrically (o.g.), on day 0 (Sigma Aldrich; $2.0 \mathrm{mg}$ in $0.2 \mathrm{ml}$ sterile water). On day 3 , the animals in the test groups received a C. difficile VPI 10463 spore challenge (o.g.; approximately $10^{3}$ colony forming units in $0.2 \mathrm{ml}$ DMEM). Animals in the treatment groups were administered $0.75 \mathrm{ml}$ of the appropriate

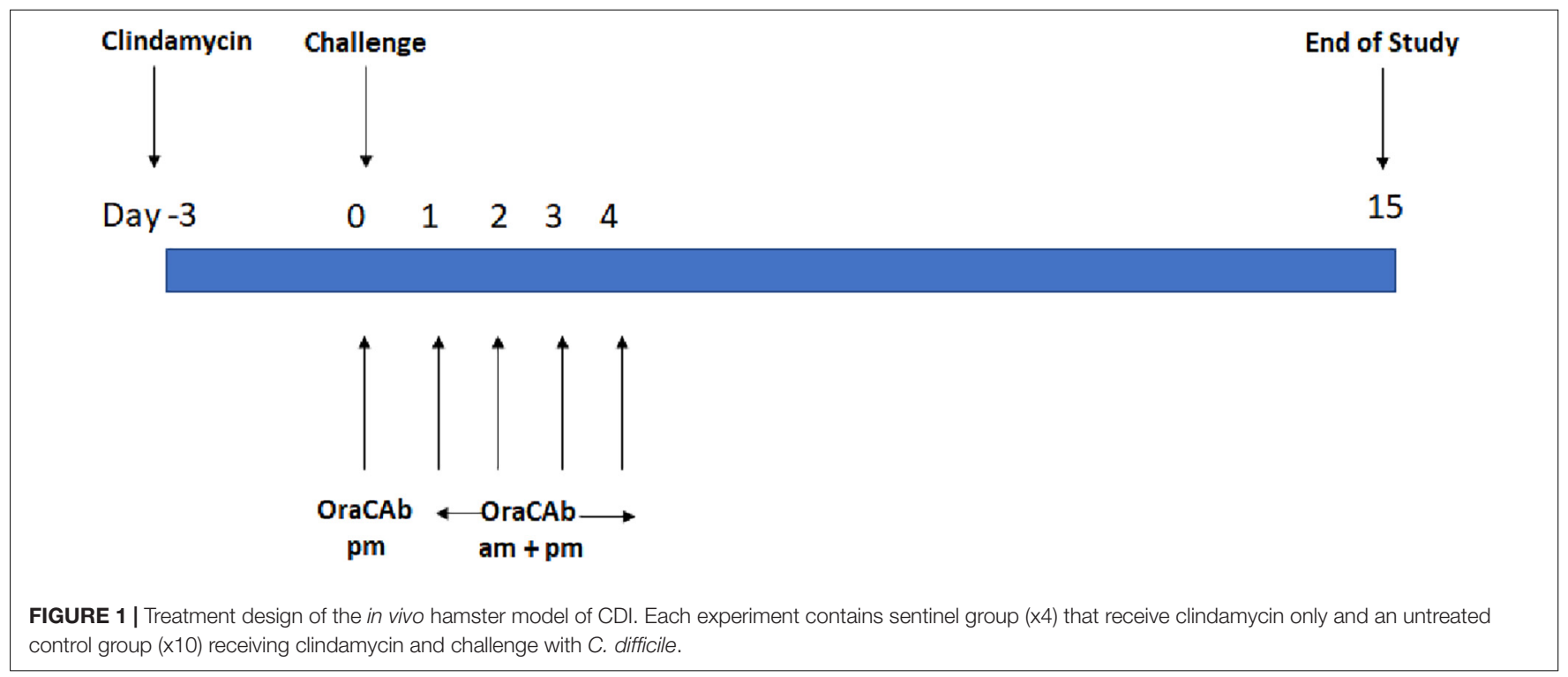


OraCAb formulation $9 \mathrm{hrs}$ post-challenge, using 18-gauge $76 \mathrm{~mm}$ metal feeding tubes (Linton Instruments). For the following 4 days, these animals received OraCAb treatment twice a day $(0.75 \mathrm{ml} /$ dose; $9 \mathrm{~h}$ interval between doses). A sentinel group (4 animals) received clindamycin $\mathrm{HCl}$, but no $C$. difficile spore challenge and acted as a cross-infection control for the experiment. Animals were not fasten prior dosing as per the Home Office license under which this experiment was carried out and they were fed ad libitum that would ensure that OraCAb encounters similar conditions during gut transit at each dosing.

The animals were weighed daily and monitored 6 times in a $24 \mathrm{~h}$ period for symptoms of CDI (diarrhoea, weight loss, lethargy, tender abdomen, activity). Animals in advanced stages of disease were euthanized. When surviving animals were symptomless for 3 days, they continued to be weighed daily and monitored twice daily.

Four different animal model studies were performed. Study A consisted of four test groups of 10 animals; (1) Untreated, (2) Received $150 \mathrm{mg} / \mathrm{mL}$ OraCAb formulation containing 3:1 antiTxB4 IgG (112.5 mg/ml): anti-TxA4 IgG (37.5 mg/ml) ratio with BBI, (3) Received $150 \mathrm{mg} / \mathrm{ml}$ OraCAb formulation containing 1:1 anti-TxB4 IgG (75 mg/ml): anti-TxA4 IgG $(75 \mathrm{mg} / \mathrm{ml})$ ratio with DEW (International Egg Products UK), (4) Received $150 \mathrm{mg} / \mathrm{ml}$ OraCAb formulation containing 3:1 anti-TxB4 IgG: anti-TxA4 IgG ratio with DEW. Study B consisted of three test groups of 10 animals; (1) Untreated, (2) Received $150 \mathrm{mg} / \mathrm{ml}$ OraCAb formulation containing 3:1 anti-TxB4 IgG: anti-TxA4 IgG ratio with DEW, (3) Received $150 \mathrm{mg} / \mathrm{ml}$ OraCAb formulation containing 3:1 anti-TxB4 IgG: anti-TxA4 IgG ratio without DEW. Study C consisted of four test groups of 10 animals; (1) Untreated, (2) Received $150 \mathrm{mg} / \mathrm{mL}$ OraCAb formulation containing 3:1 anti-TxB4 IgG: anti-TxA4 IgG ratio with DEW, (3) Received $150 \mathrm{mg} / \mathrm{mL}$ OraCAb formulation containing 1:1 anti-TxB4 IgG: anti-TxA4 IgG ratio with DEW, (4) Received $112.5 \mathrm{mg} / \mathrm{mL}$ OraCAb formulation containing anti-TxB4 IgG only with DEW. Study D consisted of four test groups of 10 animals; (1) Untreated, (2) Received $50 \mathrm{mg} / \mathrm{ml}$ OraCAb formulation containing 3:1 anti-TxB4 $\operatorname{IgG}(37.5 \mathrm{mg} / \mathrm{ml})$ : antiTxA4 IgG (12.5 mg/ml) ratio with DEW, (3) Received $100 \mathrm{mg} / \mathrm{ml}$ OraCAb formulation containing 3:1 anti-TxB4 IgG $(75 \mathrm{mg} / \mathrm{ml})$ : anti-TxA4 IgG (25 mg/ml) ratio with DEW, (4) Received $150 \mathrm{mg} / \mathrm{ml}$ OraCAb formulation containing 3:1 anti-TxB4 IgG (112.5 mg/ml): anti-TxA4 IgG $(37.5 \mathrm{mg} / \mathrm{ml})$ ratio with DEW. The compositions of the formulations are summarized in Table 1.

\section{In vitro Gut Model for C. difficile Infection}

Gut model studies were performed using a three-stage continuous culture model of the colon. This model is validated against sudden death patients for assessment of the colonic environment and ecosystem (Macfarlane et al., 1998) and has been developed for use with C. difficile (Freeman et al., 2003). Three vessels were arranged in a weir cascade with each having the $\mathrm{pH}$ controlled to model the proximal (Vessel 1, pH $5.5 \pm 0.2$ ), transverse (Vessel 2, pH $6.2 \pm 0.2$ ), and distal colon (Vessel 3, $\mathrm{pH} 6.8 \pm 0.2$ ). Vessel 1 had a volume of $280 \mathrm{ml}$, and vessels 2 and $3 \mathrm{had}$ a volume of $300 \mathrm{ml}$. Temperature was maintained at $37^{\circ} \mathrm{C}$ and an anaerobic environment was retained by continuously sparging with oxygen-free nitrogen. To maintain nutrient availability, a nutrient dense medium was added to vessel 1 at a rate of $13.2 \mathrm{ml} / \mathrm{h}$ by a peristaltic pump, and any overflow from vessel 3 was collected as waste.

Four gut model experiments were performed to assess the effect of OraCAb antibodies on $C$. difficile. Models were seeded with stool samples from a minimum of three healthy individuals, aged $>60$ years, who had not received antibiotics in the previous 3 months. Stool samples were pooled, and slurries were produced in a 1:10 w/v solution with pre- reduced PBS, equal amounts of slurry were added to each vessel. The models had no further intervention within the first 14 days to allow the bacterial communities to equilibrate and stabilize. At day 14 , and day 21 , a $1 \mathrm{ml}$ aliquot of $C$. difficile spores $\left(\sim 7 \log _{10} \mathrm{cfu} / \mathrm{ml}\right.$, strain 210$)$ was added to vessel 1 of each model. To induce CDI, vessel 1 of each model was treated with clindamycin $(33.9 \mathrm{mg} / \mathrm{l}$, four times daily). It was at this point that the treatments for the models diverge (Figure 2). Model 1; treatment with the placebo (sodium citrate buffer, three times daily, 10 days) once spore germination was detected [defined by an increase in total viable counts (TVCs) compared to spore counts], Model 2; Treatment with $0.18 \mathrm{mg} / \mathrm{ml}$ anti-TxA4 IgG $+1.6 \mathrm{mg} / \mathrm{ml}$ anti-TxB4 IgG (three times daily, 10 days) once spore germination was detected, Model 3 ; treatment with placebo once spore germination was detected, then treatment with $0.18 \mathrm{mg} / \mathrm{ml}$ anti-TxA4 IgG $+1.6 \mathrm{mg} / \mathrm{ml}$ anti-TxB4 IgG (three times daily, 10 days) once high toxin was detected (cell cytotoxicity assay of three relative units), Model 4; Treatment with vancomycin (125 mg/l four times daily, 7 days), and $0.18 \mathrm{mg} / \mathrm{ml}$ anti-TxA4 IgG + $1.6 \mathrm{~g} / \mathrm{l}$ anti-TxB4 IgG (three times daily, $\sim 35$ days), once high toxin was detected.

Gut bacteria, C. difficile TVCs and spores were enumerated three times a week during steady state, and daily thereafter using selective agars for facultative and obligate anaerobes as described previously (Chilton et al., 2014a). Samples for cytotoxin detection were taken throughout the experiment and stored at $4^{\circ} \mathrm{C}$ as described previously (Chilton et al., 2014a). Samples for antimicrobial bioassay were taken post-antibiotic exposure and stored at $-20^{\circ} \mathrm{C}$ prior to processing as described previously (Chilton et al., 2014b).

\section{Statistics}

Hamster survival data at days 5 and 15 within each plot were analyzed using the Gehan-Breslow test followed by the HolmSidak method for pairwise comparison of the groups. Differences between groups were considered statistically significant when $p<0.05$.

\section{RESULTS}

\section{TcdA and TcdB Neutralization in vitro by OraCAb Formulation}

The amount of OraCAb ovine anti-TxA4 IgG and OraCAb anti-ovine TxB4 IgG required to neutralize $1.0 \mathrm{ng}$ of TcdA or $\mathrm{TcdB}$, respectively, was calculated using a Vero cell-based toxin neutralization assay. To neutralize $1 \mathrm{ng}$ of TcdA, $0.156 \mu \mathrm{g}$ of antiTxA4 IgG were sufficient. For neutralization of 1 ng of TcdB $8 \mu \mathrm{g}$ 


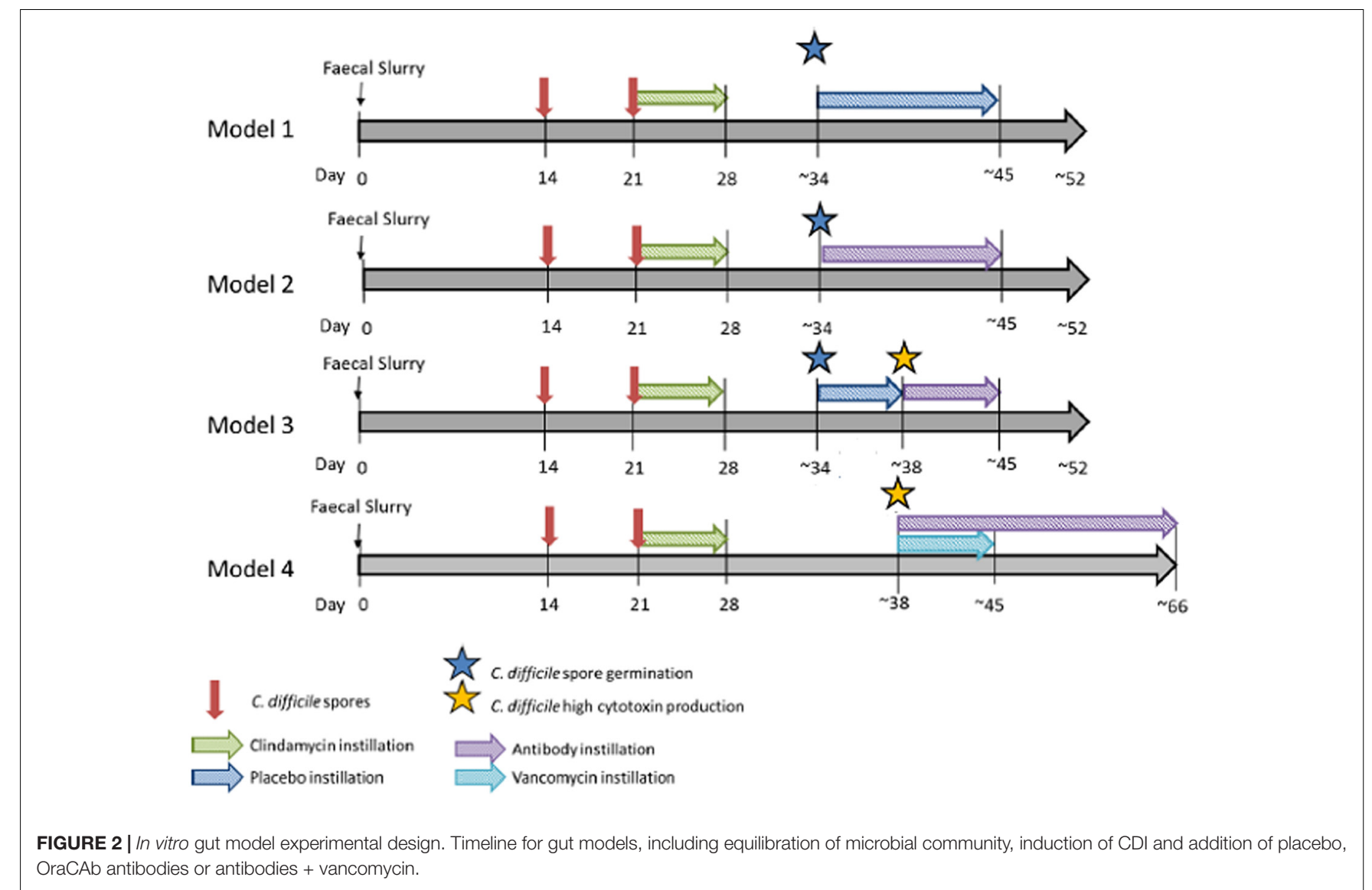

of anti-TxB4 IgG were required. The lower neutralizing capacity of antibodies to TxB4 was taken into account in the design of the protocols used in the hamster model and the in vitro gut model. Previously, we determined that antibodies to both toxins were required for protection in the hamster model when the antibodies were administered systemically (Roberts et al., 2012). However, others have shown that systemic administration of antibodies against TcdA and TcdB do not improve the efficacy of treatment of CDI compared to administration of anti-TcdB only in piglets (Steele et al., 2013) and humans (Lee et al., 2017). Considering the specificity of different CDI models, the in vivo studies described here used formulations with anti-TxB4:anti-TxA4 ratios up to $3: 1$, while for the treatment of CDI in the in vitro human gut model we used a ratio of approximately 9:1. Anti-TxA4 was not abolished completely in the human gut model as in this study antibodies were administered orally to act directly at the site of infection, thus in an in vivo setup anti-TxA4 would protect gut epithelium from further damage and prevent infiltration of antibodies in the circulation.

\section{Protection of Antibodies in the Formulation From Enzymatic Digestion in the GI Tract}

To enable effective neutralization by OraCAb, the antibodies in the formulation must be delivered intact to the large intestine.
Trypsin is the major proteolytic enzyme of the small intestine. DEW and BBI, the latter which can be purified from Lima beans, are two options that can be used as inhibitors of trypsin in OraCAb formulation to protect the integrity of the antibodies. Thus, we investigated the inhibition of human trypsin in vitro by either DEW or BBI. The BBI inhibitor was more potent, with approximately 15 -fold less BBI being required, compared to DEW, to inhibit the same amount of human trypsin (Figure 3). This data together with available information on concentration of trypsin in human GI tract (Borgstrom et al., 1957) were used to inform levels of the two inhibitors to be included in the OraCAb formulations used in the in vivo experiments.

\section{Protection of Antibodies in the Formulation From Acid pH}

The low $\mathrm{pH}$ in human gastric fluid (1.5-3.5) and the presence of pepsin, would inactivate the proteins in the OraCAb formulation by affecting their conformation and integrity. OraCAb formulation contains antacids (magnesium hydroxide and aluminum hydroxide), which have been used widely in medicines for the treatment of dyspepsia, to protect the antibodies and protease inhibitors from inactivation in the stomach. The presence of antacids will also raise the stomach $\mathrm{pH}$ leading to inactivation of pepsin. The antacids neutralize the $\mathrm{pH}$ in the stomach once the OraCAb is taken. The aluminum 


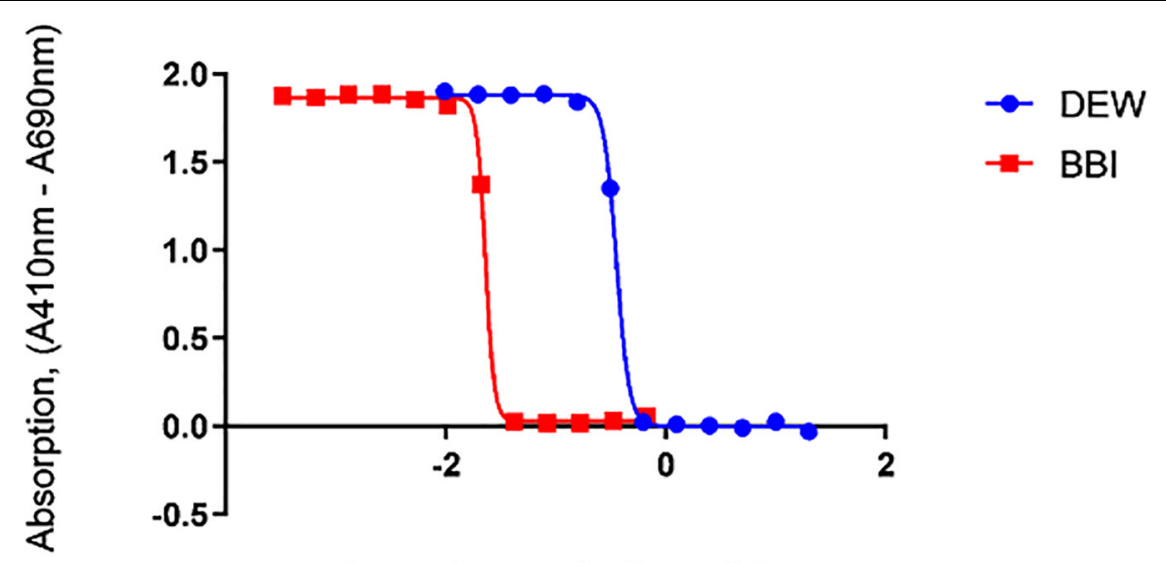

$\log _{10}$ (concentration, g/L)

\begin{tabular}{|l|c|}
\cline { 2 - 2 } \multicolumn{1}{c|}{} & EC50, $\mathrm{mg} / \mathrm{L}$ \\
\hline DEW & 355.2 \\
\hline BBI & 22.9 \\
\hline
\end{tabular}

FIGURE 3 | Inhibition of human trypsin with DEW and purified BBI determined by Na-Benzoyl-DL-Arginine-p-Nitroanilide (BAPNA) colorimetric assay. Concentrations of DEW and $\mathrm{BBI}$ that gave half-maximal inhibition of human trypsin $\left(E_{50}\right)$ are given in the table.

hydroxide gel is also needed to maintain the higher $\mathrm{pH}$ while the drug is present within the stomach and additional gastric fluids are released. Antacid concentrations in OraCAb provide a similar daily intake as that from dyspepsia medications. We studied the dose of OraCAb that needed to be administered orally to ensure full protection of the IgG in a simulated gastric fluid (SGF). The data (Figure 4) show that OraCAb mixed with the $S G F$ at $3 / 10 \mathrm{v} / \mathrm{v}$ ratio was sufficient to fully preserve the activity of the IgG. An example of the protection of anti-TxA4 IgG in OraCAb from SGF is given in Figure 4.

\section{A Trypsin Inhibitor Appears to Preserve OraCAb Activity in vivo}

To determine whether OraCAb toxin neutralization can prevent CDI in vivo, we used the Syrian hamster model. Animals were given clindamycin orally 3 days before challenge and then challenged with $C$. difficile spores via the oral route on day 0 . An untreated group acted as controls and a sentinel group that received clindamycin, but no challenge, was included to check for cross-contamination. Test groups contained 10 animals and sentinel groups consisted of four animals. The animals were then placed on one of the oral therapeutic regimes until the end of day 4 (Table 1) and monitored until day 15 . Statistical analyses were performed on day 5 (end of treatment) and day 15 (end of the study). Survival results for each study are shown in Figure $\mathbf{5}$ and Table 2.

We investigated the effects of inclusion of two different protease inhibitors in the OraCAb formulation, BBI $(10 \mathrm{mg} / \mathrm{ml})$ and DEW (60 mg/ml) (Figure 5). In study A, no significant difference was observed between groups given formulations containing DEW inhibitor and those receiving a formulation containing BBI trypsin inhibitor ( $p=1.0$, Figure 5A), indicating the benefit of these two inhibitors to be comparable in the hamster model. In study $\mathrm{B}$, disease progression was rapid with no untreated animals surviving to the second day post challenge. On day four the survival rate in the group treated with OraCAb containing $\mathrm{DEW}$ was $50 \%$, in the absence of protease inhibitor it was $10 \%$ (Figure 5B). By day ten there were no survivors in either group. In this study there was a significant difference between the survival of animals given OraCAb containing DEW and those treated with OraCAb where DEW was omitted $(p=0.021)$ and a clinical benefit of the addition of DEW can be observed during the treatment period.

\section{OraCAb Formulations Containing Antibodies to Both TcdA and TcdB Protect Hamsters From CDI}

During the production and assessment of the ovine antibodies to TxA4 and TxB4, we noted that the toxin neutralizing capacity of ovine anti-TxB4 IgG was lower than that of ovine anti-TxA4 IgG. In addition, in previous studies we determined that antibodies to both toxins were required for protection in the hamster model when the antibodies were administered systemically (Roberts et al., 2012). Therefore, we assessed the efficacy of different OraCAb formulations containing different ratios of antiTxB4: anti-TxA4 IgG, as well as a formulation containing anti-TxB4 IgG only.

All animals that received an OraCAb formulation containing both anti-TxB4 and anti-TxA4 antibodies had a significantly 

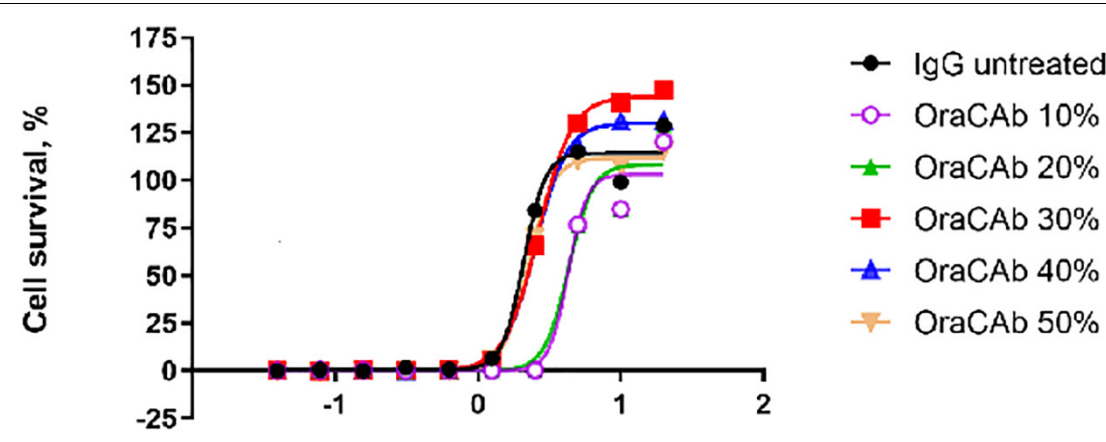

$\log _{10}$ (IgG concentration, $\mathbf{m g} / \mathrm{L}$ )

\begin{tabular}{|c|c|c|c|c|c|c|}
\cline { 2 - 7 } \multicolumn{1}{c|}{} & $\begin{array}{c}\text { IgG, } \\
\text { untreated }\end{array}$ & $\begin{array}{c}\text { OraCAb, } \\
10 \%\end{array}$ & $\begin{array}{c}\text { OraCAb, } \\
20 \%\end{array}$ & $\begin{array}{c}\text { OraCAb, } \\
30 \%\end{array}$ & $\begin{array}{c}\text { OraCAb, } \\
40 \%\end{array}$ & $\begin{array}{c}\text { OraCAb, } \\
50 \%\end{array}$ \\
\hline EC50, mg/L & 0.3205 & 0.6323 & 0.6359 & 0.4226 & 0.4011 & 0.3512 \\
\hline
\end{tabular}

FIGURE 4 | Stability of IgG (anti-TxA4) in OraCAb formulation in SGF. OraCAb was incubated for $2.5 \mathrm{~h}$ in SGF at different v/v ratios and full protection of the IgG was found when the volume of OraCAb was 30\% (3/10 ratio) or higher compared with the volume of the SGF. Around 50\% of the IgG was found inactivated/degraded in SGF containing 10 or $20 \%$ OraCAb. Concentrations of IgG in OraCAb that give half maximum inhibition of TcdA in a cell-based toxin neutralization assay (EC 50 ) are given in the table. The calculations were based on the concentration of IgG prior to mixing the formulations with SGF.

higher survival rate compared to untreated animals, whereas those receiving anti-TxB4 $\mathrm{IgG}$ only did not (Figure 5C). In study C (Figure 5C), 80\% of the untreated animals succumbed to CDI 5 days post-challenge. During this time interval, all animals treated with OraCAb containing antibodies to both toxins remained healthy. However, $40 \%$ of the group receiving anti-TxB4 IgG only had succumbed to CDI. There was a significant difference between the survival rates of animals receiving 3:1 anti-TxB4: anti-TxA4 IgG and 1:1 anti-TxB4: antiTxA4 IgG compared to the control group ( $p=0.018$ and 0.049 , respectively). Animals receiving OraCAb containing antibodies that neutralize $\mathrm{TcdB}$ only did not experience a statistically meaningful clinical benefit over untreated animals $(p=0.30)$. In study A (Figure 5A), all untreated animals succumbed to CDI by day 5 post-challenge, in contrast to animals receiving OraCAb, where all animals were protected from CDI during this period. Fifty percent of the animals receiving OraCAb with 1:1 anti-TxB4 to anti-TxA4 IgG ratio and $80 \%$ of animals receiving OraCAb with $3: 1$ anti-TxB4 to anti-TxA4 IgG ratio were protected and survived to the end of the study $(p<0.0001$ compared to the untreated group). All OraCAb formulations produced a significant clinical benefit relative to the untreated control group. OraCAb, containing antibodies that neutralize both TcdA and TcdB, appears to be protective in the hamster model of CDI. The presence of antibodies to TcdA in the gut appears to give increased efficacy of OraCAb in the hamster model of CDI. A direct ELISA for detection of infiltrated ovine IgGs in the sera from hamsters with CDI and treated with OraCAb was developed (see Supplementary Material). No presence of ovine IgG was detected in any of the tested 15 sera (Supplementary Figure S2). Although the sera samples were collected 10 days after the final OraCAb treatment, the high sensitivity of the ELISA and the long half-life of ovine IgGs (more than 10 days, Watson, 1992) strongly support the conclusion that there was no significant systemic infiltration of ovine IgGs in hamsters with CDI.

\section{OraCAb Formulations Containing 3:1 Ratio of Anti-TxB4 to Anti-TxA4 IgG May Be Superior to Those Containing 1:1 Ratios}

For both studies A and C (Figures 5A,C), a higher survival rate was observed for animals receiving the 3:1 anti-TxB4 to antiTxA4 IgG OraCAb formulation compared with those receiving the $1: 1$ antibody formulation. In study $A, 80 \%$ of the animals receiving the 3:1 formulation survived to the end of the study compared to $50 \%$ given the $1: 1$ ratio OraCAb. For study C, percentage survival was 60 and $20 \%$ for the $3: 1$ ratio and the 1:1 ratio, respectively. Although survival data between the two antibody ratios did not reach statistical significance in either study, the data generated from both studies indicates a better survival outcome and clinical benefit in the hamster model when animals were treated with the OraCAb formulation with a ratio of 3:1 anti-TxB4 to anti-TxA4 IgG. The statistical method used to assess significance between different treatment groups is based on the area under the survival curves. Thus, although a difference in the survival rate of these two groups was observed after the end of the treatment, significance between these two groups was not achieved most likely due to their comparable outcome during the treatment period.

\section{OraCAb Administration Protects Hamsters in a Dose-Dependent Manner}

We investigated the dose dependency of OraCAb using three different concentrations of antibody in a 3:1 ratio of anti-TxB4:anti-TxA4 IgG. In study D, CDI progression was rapid with no untreated animals surviving to the 
A

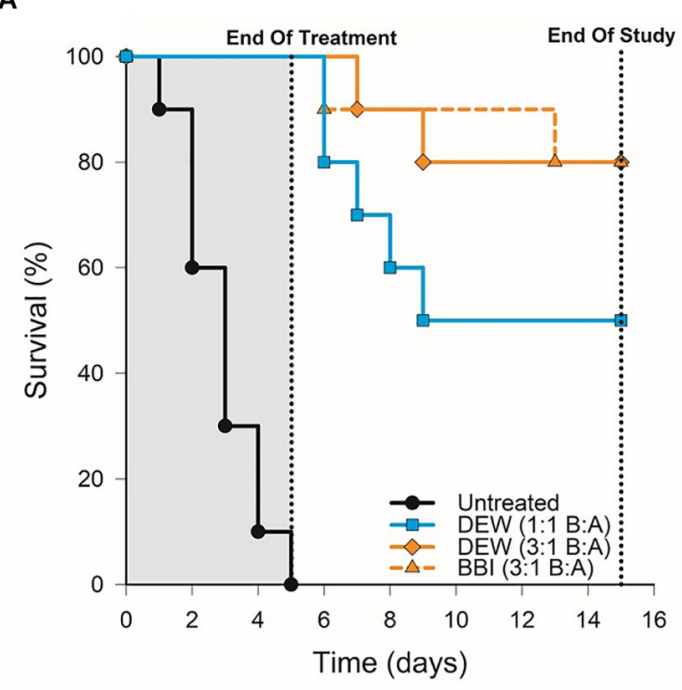

C

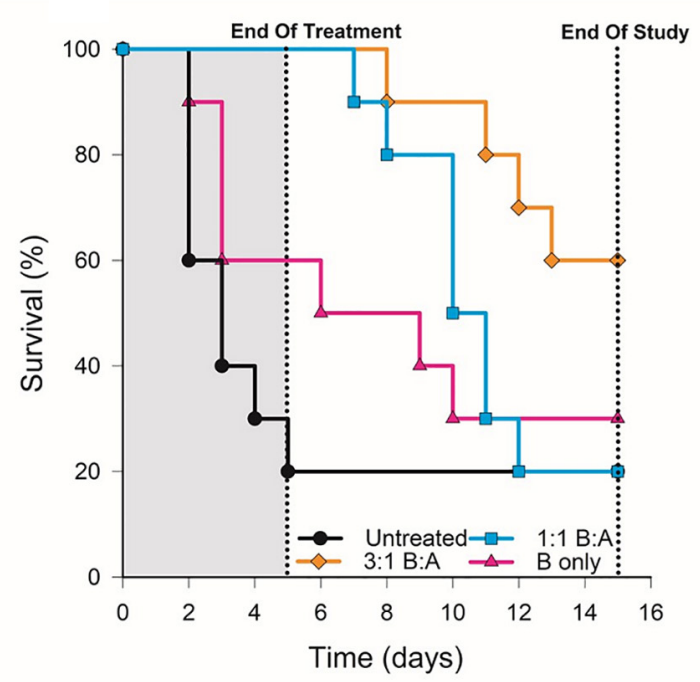

B

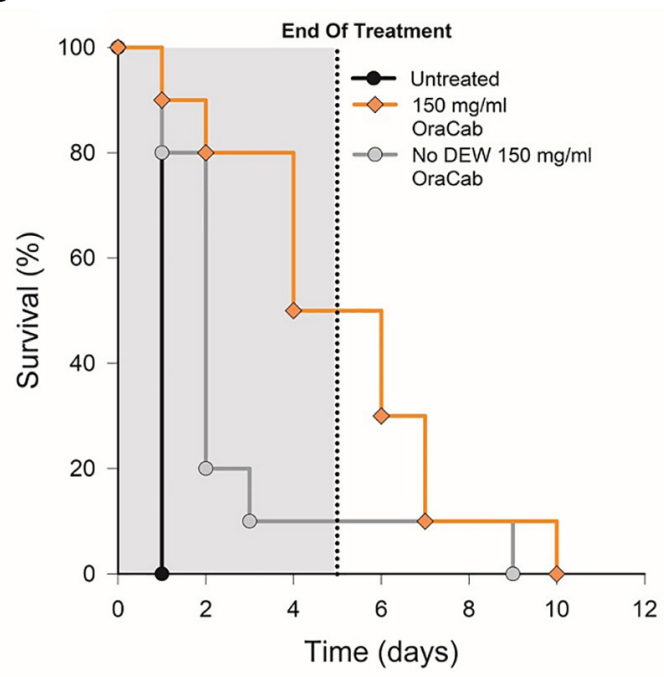

D

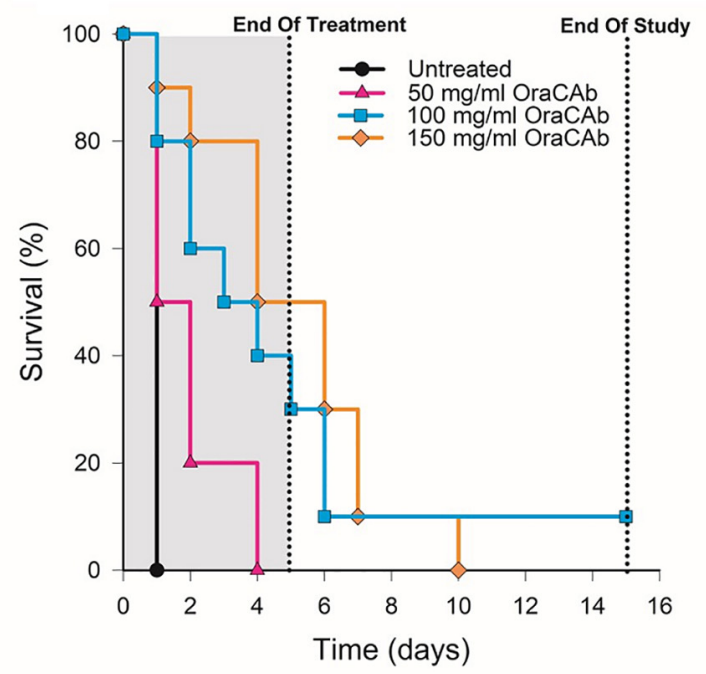

FIGURE 5 | Survival rates of hamsters after $C$. difficile challenge. Hamsters were challenged with $C$. difficile spores via the oral route on day 0 , placed on one of the oral therapeutic regimes until the end of day 4 and monitored until day 15. An untreated control group, receiving challenge only, was included for each experiment. (A) Groups were treated with OraCAb containing different ratios of anti-TxB4: anti-TxA4 antibodies with either BBI or DEW as trypsin inhibitors. (B) Groups were treated using OraCAb formulated with antibody concentration of $150 \mathrm{mg} / \mathrm{ml}$ at a 3:1 anti-TxB4: anti-TxA4 ratio in the presence or absence of DEW. (C) Groups were treated with OraCAb containing different ratios of anti-TxB4: anti-TxA4 antibodies. The antibodies in OraCAb that contained anti-TxB4 IgG only were at the same concentration as anti-TxB4 antibodies in the 3:1 anti-TxB4: anti-TxA4 formulation. All OraCAb formulations contained DEW. (D) Groups were treated with OraCAb containing DEW and three different antibody concentrations (50, 100, or $150 \mathrm{mg} / \mathrm{ml})$ at a fixed 3:1 anti-TxB4: anti-TxA4 ratio. The table shows the outcome of the statistical analyses of the data.

second day post challenge (Figure 5D). Compared to the untreated group, all OraCAb formulations provided a significant increase in survival rate $(50 \mathrm{mg} / \mathrm{ml} p=0.035$, $100 \mathrm{mg} / \mathrm{ml} p=0.002,150 \mathrm{mg} / \mathrm{ml} p<0.0005)$. The $150 \mathrm{mg} / \mathrm{ml}$ antibody OraCAb provided significantly better clinical benefit compared to the $50 \mathrm{mg} / \mathrm{ml}$ antibody OraCAb formulation $(p=0.016)$. No statistically meaningful differences were observed between treatment groups receiving $100 \mathrm{mg} / \mathrm{ml}$ and $150 \mathrm{mg} / \mathrm{ml}$ antibody OraCAb formulations $(p=0.30)$. The data suggest that OraCAb containing greater than
$50 \mathrm{mg} / \mathrm{ml} \mathrm{IgG}$ is required for protection from CDI in the hamster model.

\section{OraCAb Antibody Instillation Prevents Toxin Detection in an in vitro Gut Model of CDI}

A well validated and clinically reflective in vitro gut model of CDI was used to investigate the efficacy of OraCAb instillation in the presence of the normal human microbiota. The models were 
TABLE 2 | $P$-values calculated for different treatments in the in vivo model of CDI.

\begin{tabular}{|c|c|c|c|}
\hline Study & Comparison & $P$-value end of study & $P$-value end of treatment \\
\hline \multirow[t]{4}{*}{ A } & OraCAb DEW 3:1 B:A vs. untreated & $<0.001$ & $<0.001$ \\
\hline & OraCAb BBI 3:1 B:A vs. untreated & $<0.001$ & $<0.001$ \\
\hline & OraCAb DEW 1:1 B:A vs. untreated & $<0.001$ & $<0.001$ \\
\hline & OraCAb DEW 3:1 B:A vs. OraCAb BBI 3:1 B:A & 1.0 & 1.0 \\
\hline \multirow[t]{3}{*}{$B$} & OraCAb DEW 3:1 B:A vs. untreated & 0.008 & $<0.001$ \\
\hline & OraCAb No DEW 3:1 B:A vs. untreated & 0.003 & 0.001 \\
\hline & OraCAb DEW 3:1 B:A vs. OraCAb No DEW 3:1 B:A & 0.021 & 0.014 \\
\hline \multirow[t]{3}{*}{ C } & OraCAb DEW 3:1 B:A vs. untreated & 0.018 & 0.003 \\
\hline & OraCAb DEW 1:1 B:A vs. untreated & 0.049 & 0.002 \\
\hline & OraCAb DEW anti-TxB4 only vs. untreated & 0.30 & 0.18 \\
\hline \multirow[t]{5}{*}{$\mathrm{D}$} & OraCAb 50 mg/ml lgG vs. untreated & 0.035 & 0.004 \\
\hline & OraCAb 100 mg/ml lgG vs. untreated & 0.002 & 0.002 \\
\hline & OraCAb 150 mg/ml lgG vs. untreated & 0.008 & $<0.001$ \\
\hline & OraCAb 50 mg/ml lgG vs. OraCAb 150 mg/ml lgG & 0.016 & 0.016 \\
\hline & OraCAb 150 mg/ml lgG vs. OraCAb 100 mg/ml lgG & 0.3 & 0.3 \\
\hline
\end{tabular}

populated with a pooled fecal slurry and left to equilibrate before simulated CDI was induced using clindamycin. Models were treated with either (A) placebo (sodium citrate saline buffer), (B) OraCAb antibodies alone, (C) placebo followed by OraCAb antibodies (D) OraCAb antibodies alongside standard of care (vancomycin). The OraCAb antibodies were formulated as 9:1 anti-TxB4 IgG: anti-TxA4 IgG in a sodium citrate saline buffer. One of the benefits of using an in vitro system such as the gut model in pre-clinical evaluation is that longitudinal trends (such as $C$. difficile spore germination and toxin production) can be followed in a way that is not possible clinically. In models A and $B$ it was specifically chosen to start instillation at the point of spore germination in order to assess the impact of OraCAb as an early intervention (to reflect the use of OraCAb as prophylaxis, e.g., in prevention of recurrence). In Model C, it was instilled from toxin detection (simulating the point at which a patient was symptomatic), and in Model D alongside the 'standard of care' vancomycin. Thus we were able to gain data on the potential efficacy of OraCAb administered in a variety of clinical scenarios.

Clindamycin exposure induced simulated CDI in all models (Figure 6). Simulated CDI is defined by an increase in TVCs over spore counts (indicating $C$. difficile spore germination and vegetative cell proliferation), with concomitant high cytotoxin detection. Instillation of placebo did not prevent $C$. difficile vegetative growth or toxin detection (Figure 6A). TVCs peaked at $\sim 6.5 \log _{10} \mathrm{cfu} / \mathrm{ml}$ (spore counts $\sim 2 \log _{10} \mathrm{cfu} / \mathrm{ml}$ ) and a peak toxin titer of 3 relative units was observed. Both C. difficile vegetative growth and toxin production reduced, before increasing again at the end of the experiment. Instillation of OraCAb antibodies at the first sign of $C$. difficile spore germination (increasing TVC over spore counts) did not affect C. difficile vegetative growth (TVCs peaked at $\sim 6 \log _{10} \mathrm{cfu} / \mathrm{ml}$ with spores $\sim 2.5 \log _{10} \mathrm{cfu} / \mathrm{ml}$ ), but completely prevented the detection of $C$. difficile cytotoxin (Figure 6B). Instillation of placebo at the first sign of spore germination followed by instillation of OraCAb antibodies at simulated CDI (high toxin detection) led to a rapid decrease in detectable toxin (2 days after peak toxin compared to 5 days after peak toxin in the placebo treated model). No further toxin was detected during OraCAb antibody instillation, apart from a low level titer of 1 on the final day of experiment. This was despite a second increase in TVC counts compared to spores, indicating prolonged vegetative cell proliferation (Figure 6C).

\section{OraCAb Antibody Instillation Alongside Standard of Care (Vancomycin) Prevents Simulated Recurrent CDI in an in vitro Gut Model of CDI}

Instillation of OraCAb antibodies alongside vancomycin caused rapid reduction in $C$. difficile vegetative cells and cytotoxin levels, as has been observed previously when vancomycin is used to treat simulated CDI in the gut models (Freeman et al., 2005; Baines et al., 2008; Chilton et al., 2012, 2014b; Crowther et al., 2015). No further toxin was detected throughout the experiment (Figure 6D). Importantly, in model 4 (Figure 6D), recurrent C. difficile vegetative cell growth was observed post vancomycin instillation. It would be expected that this would be accompanied by cytotoxin production (simulated recurrent disease) as has been observed previously (Chilton et al., 2012, 2014b; Crowther et al., 2015), however, with the installation of the OraCAb antibodies, no toxin was detected.

\section{OraCAb Antibody Instillation Did Not Affect Gut Microbiota Populations in an in vitro Gut Model of CDI}

Instillation of antibodies did not affect microbiota populations, the dynamics of which were similar in all experiments and 
A

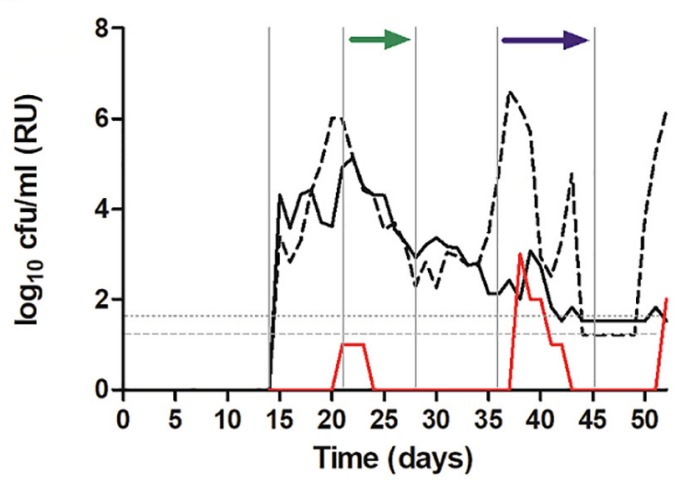

C

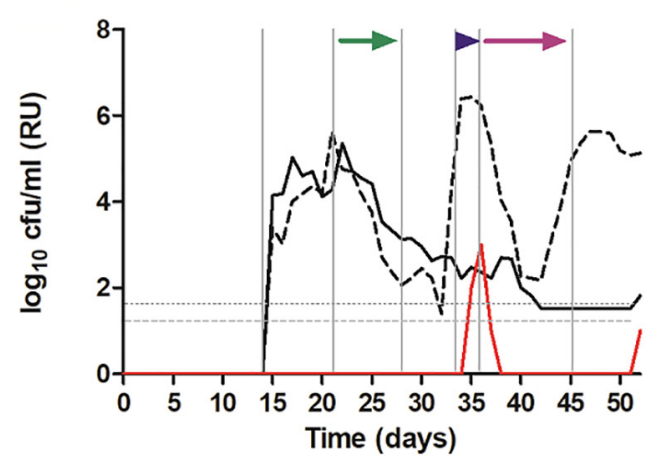

B

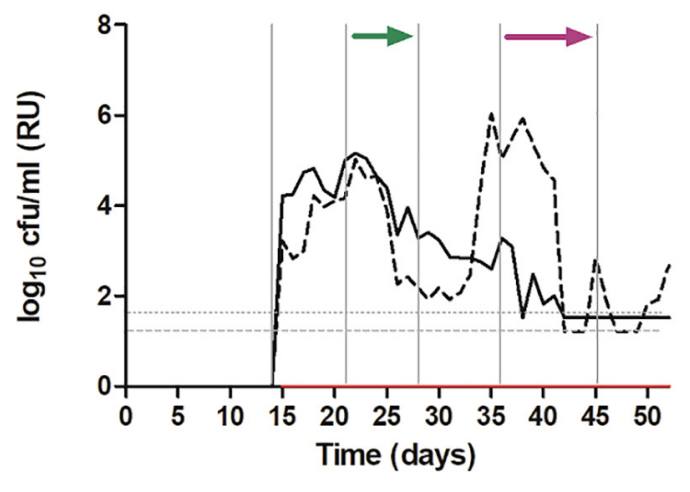

D
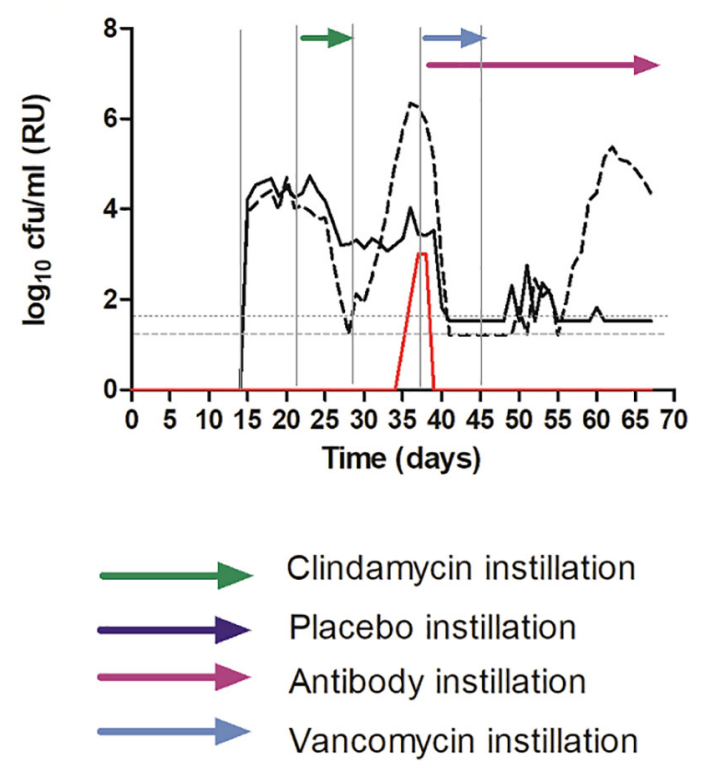

FIGURE 6 | Mean C. difficile total viable counts ( $\left.\log _{10} \mathrm{cfu} / \mathrm{ml}\right)$, spore counts $\left(\log _{10} \mathrm{cfu} / \mathrm{ml}\right)$ and cytotoxin titer [relative units (RU)] in vessel three of the in vitro gut model. (A) - Model 1 (placebo); (B) - Model 2 (antibodies); (C) - Model 3 (placebo + antibodies); (D) - Model 4 (vancomycin + antibodies). The limit of detection is $1.22 \log _{10} \mathrm{cfu} / \mathrm{ml}$ for total viable counts and $1.52 \log _{10} \mathrm{cfu} / \mathrm{ml}$ for spore counts; horizontal dotted lines.

to those from previously reported in vitro gut model studies (Freeman et al., 2005; Baines et al., 2008; Chilton et al., 2012, 2014a; Crowther et al., 2015). The results from models 1 and 2, receiving placebo and antibody are shown here (Figure 7).

Although some variation was observed between individual models, clindamycin instillation had similar effects to those reported previously in the in vitro gut model of CDI, causing declines in Bifidobacterium species $\left(\sim 6 \log _{10} \mathrm{cfu} / \mathrm{ml}\right.$ to below the limit of detection), and Lactobacillus populations $\left(\sim 2-4 \log _{10}\right.$ $\mathrm{cfu} / \mathrm{ml})$, and increases in lactose fermenting Enterobacteriaceae $\left(\sim 2 \log _{10} \mathrm{cfu} / \mathrm{ml}\right)$ and Enterococcus species $\left(\sim 4 \log _{10} \mathrm{cfu} / \mathrm{ml}\right)$. Recovery following clindamycin exposure was similar regardless of whether models were exposed to antibodies or placebo. These data provide an important indication that the effects of OraCAb on humanized gut microbiota is minimal.

These results highlight the protective capacity provided by an orally delivered ovine polyclonal antibody formulation designed to neutralize both $\mathrm{TcdA}$ and TcdB. The formulation protects hamsters from CDI and the antibodies have been shown to neutralize TcdA and TcdB in the human gut model. The antibodies can be used alongside standard of care antibiotics and they do not have a deleterious effect on the microbial flora.

\section{DISCUSSION}

CDI remains a problem within healthcare systems of the developed (Lessa et al., 2015) and the developing world (Chaudhry et al., 2017). It is interesting that at present approximately $60 \%$ of CDI cases are defined as 'communityacquired,' but this leads to further cases needing hospitalization (Ofori et al., 2018; Public Health England, 2019). Even with effective therapy, recurrent infection is common, ranging from $20 \%$ after initial infection to $60 \%$ after multiple prior recurrences 

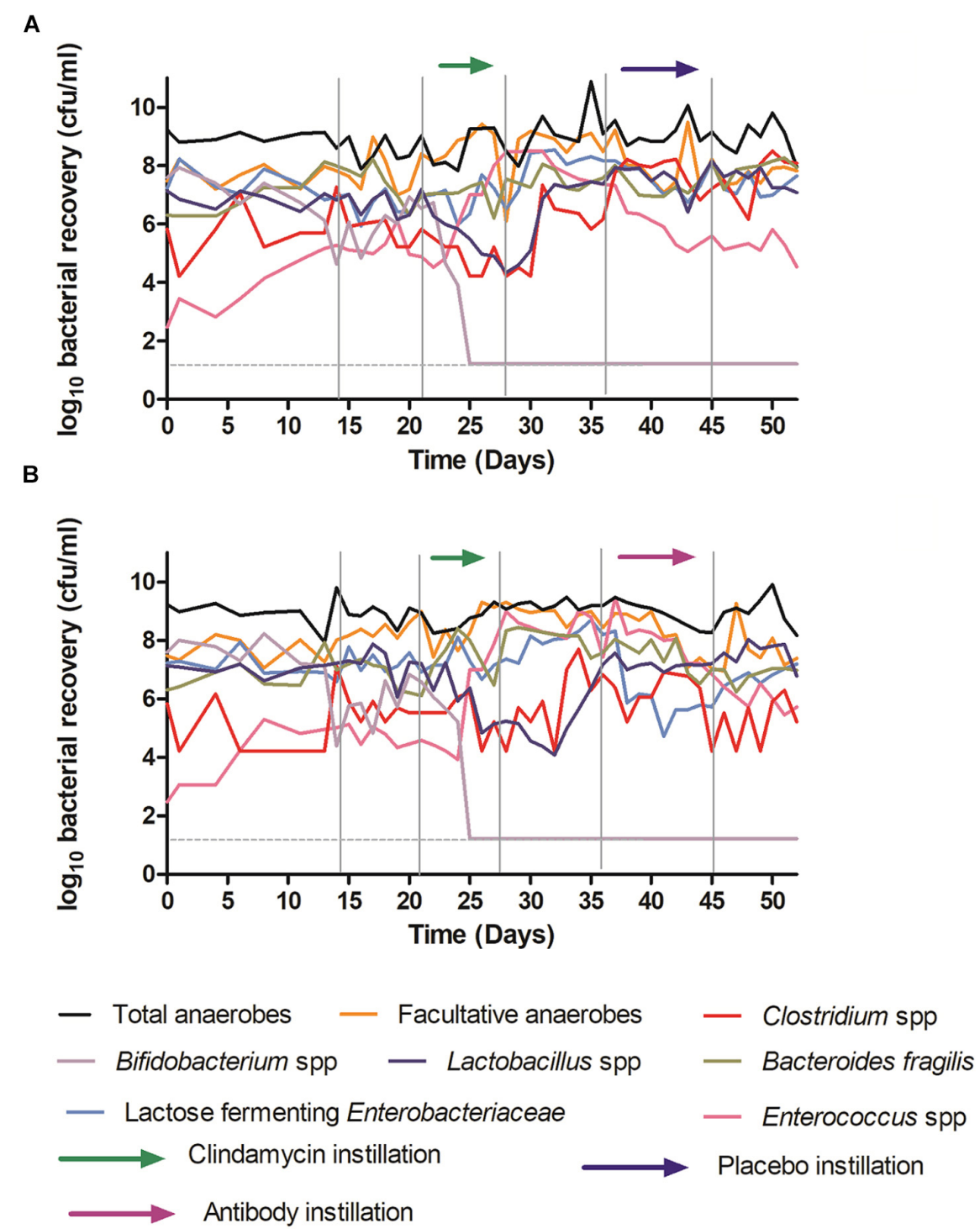

FIGURE 7 | Mean viable counts of selected microbiota communities in vessel three of the in vitro gut model. (A) - Model 1 (placebo); (B) - Model 2 (antibodies). The limit of detection is $1.22 \log _{10} \mathrm{cfu} / \mathrm{ml}$; horizontal dotted line.

(McFarland et al., 1999), suggesting that antibiotic treatment has failed to eradicate $C$. difficile and/or exacerbated the dysbiosis that initially allowed the bacterium to proliferate and produce toxin. Therefore, additional therapeutic options are required for use when antibiotic treatment alone fails (Jawa and Mercer, 2012). For example, a combined standard of care antibiotic and systemically administered bezlotoxumab, a MAb against TcdB, has been proved to be clinically effective in the prevention of recurrent CDI (Wilcox et al., 2017).

Previously we described development of a therapeutic for CDI based on the administration of ovine polyclonal antibodies to novel, non-toxic recombinant antigens, TxA4 and TxB4, based on TcdA and TcdB. This was the first use of ovine serum antibodies in the treatment of CDI. These antibodies potently neutralize TcdA and TcdB in both in vitro cell assays and in an in vivo hamster model for CDI (Maynard-Smith et al., 2014). In the present study we show that these ovine antibodies can also be formulated for oral delivery and can act as an effective therapeutic for CDI. We also show in an in vitro human gut model that the antibodies prevent the detection of toxin activity during simulated recurrent CDI. Unlike antibiotics, OraCAb antibodies do not have a deleterious effect on the human gut flora. This is the first study to describe the use of orally delivered ovine polyclonal IgG for the treatment of CDI.

To our knowledge, this is the first use of an in vitro gut model seeded with a human fecal inoculum to test the effects of oral antibodies for $C$. difficile infection. Treatment with clindamycin and vancomycin, resulted in a loss of colonization resistance and led to CDI, as previously identified in gut model studies (Chilton et al., 2014a; Crowther et al., 2015). OraCAb repeatedly 
neutralized $C$. difficile cytotoxin, Figure $6 \mathrm{C}$ demonstrating that within $48 \mathrm{~h}$ the toxin produced was neutralized from a titer of 3 RU to undetectable. Additionally, consistent treatment with OraCAb, prevented the detection of $C$. difficile cytotoxin for greater than $48 \mathrm{~h}$ (Figures 5B-D). Of note, although OraCAb prevented the detection of cytotoxin, it did not prevent the growth and spore germination of $C$. difficile itself. OraCAb has been developed to reduce the toxin burden in the gut and thus it was not expected to have anti-C. difficile properties. As CDI is a toxin mediated disease (Lyerly et al., 1985), these results suggest that OraCAb would be beneficial, as a potential prophylaxic, alongside standard of care treatments for CDI.

Passive immunization, using both polyclonal (mostly of bovine colostrum and chicken egg origin) and MAbs, to protect against CDI has been studied extensively in animal models (Kink and Williams, 1998; Roberts et al., 2012; Maynard-Smith et al., 2014; Sponseller et al., 2015; Pizarro-Guajardo et al., 2017). Hyperimmune ovine serum, unlike bovine colostrum, can be continually supplied and requires only immunization boosts at certain time intervals. Polyclonal antibodies have several advantages over MAbs. Firstly, they are cheaper to produce, and secondly, they bind to several epitopes on their antigen, increasing the neutralizing efficacy of the therapeutic against sequence variants, such as those observed in TcdA and TcdB of $C$. difficile. Recently, Cole and co-workers demonstrated that polyclonal sera to both TcdA and TcdB when tested in an in vitro toxin neutralization assay, displayed greater activity against the toxins than equivalent concentrations of individual MAbs (Cole et al., 2019). Animal-derived polyclonal antibodies have the disadvantage of inducing an immune response in the recipient when delivered systemically and thus the number of doses will be limited. In severe disease, where therapeutic options have been exhausted, one or two doses may be all that is required to alleviate symptoms. Recently, bezlotoxumab, a MAb-based therapy, has been licensed for use in the prevention of recurrent CDI (Wilcox et al., 2017). However, CDI is an infection of the large intestine where the virulence factors act on the intestinal epithelium and therefore the oral delivery of an immunotherapeutic at the site of toxin production and action would be advantageous. Oral administration would be the preferable route for nonsevere and/or recurrent CDI allowing repeated treatment to take place due to lack of adverse humoral responses in circulation as indicated by clinical trials with orally delivered polyclonal antibodies against TNF $\alpha$ (AVX-470) for treatment of ulcerative colitis (Harris et al., 2016), a disease which is also associated with severe colon inflammation. Zhang et al. (2015) have argued that the requirement for epithelial disruption to allow transport of systemic antibodies to the site of infection (i.e., the gut lumen) is actually an advantage as it provides a constant source of antibodies over time to defend against future infections. Indeed, this is correct in severe CDI when systemic administration of antibodies against $C$. difficile toxins would have no alternative.

There have been a few clinical trials in which bovine antibodies have been administered orally in healthy or challenged individuals (Jasion and Burnett, 2015). Data from these trials show survival of orally administered immunoglobulins through the digestive tract in humans to be between 0.01 and $20 \%$. These observations along with our data that addition of a protease inhibitor to the formulation led to significant improvement in survival of animals during the treatment (Figure 5B) clearly demonstrate the need for protection of immunoglobulins from digestion and/or inactivation in the GI tract. The OraCAb formulation that we report here protects the proteins in this formulation from degradation/inactivation by low $\mathrm{pH}$ in gastric fluid and from proteases during transit from oral administration to the large intestine (Figures 2, 3). Two protease inhibitors were investigated for inclusion in OraCAb. BBI is a highly purified trypsin and chymotrypsin inhibitor from Lima beans. It can pass through the stomach and small intestine without major degradation (Clemente et al., 2011). DEW contains several protease inhibitors (Saxena and Tayyab, 1997). It is known that some proteolytic inhibitors have a feedback control of proteolytic enzyme secretion in humans (Liener et al., 1986) and also in animals, e.g., rats (Geratz and Hurt, 1970). Some trypsin/chymotrypsin inhibitors stimulate secretion of proteolytic enzymes into small intestines (e.g., Bowman-Birk Inhibitor, 4,4'-diamino-diphenylamine), however, others like $p$-aminobenzamidine does not seem to have significant effect on secretion of trypsin and chymotrypsin in small intestine in rats (Geratz and Hurt, 1970). DEW has not been tested for effect on secretion of proteases in small intestines. However, the quantity of trypsin/chymotrypsin inhibitors in OraCAb has been calculated based on data for concentrations of these two enzymes in human duodenum during digestion of standard meal (Worning and Müllertz, 1966) with an excess to allow for possible increased secretion of these proteases. Our in vivo data (Figure 5A) showed that the 2.5-fold higher potency of BBI in the formulation compared to that of DEW did not make significant difference in survival of the hamsters. The fact that increasing the potency of trypsin/chymotrypsin inhibitors with a feedback control did not affect the survival rates demonstrate that the concentration of $60 \mathrm{mg} / \mathrm{ml} \mathrm{DEW}$ was sufficient to ensure protection from proteolytic enzymes in the hamster model of CDI even if a similar feedback control in release of proteolytic enzymes takes place in presence of DEW. Thus, subsequent experiments were performed with OraCAb containing DEW which considerably reduces the manufacture cost of OraCAb.

Over recent years there has been much conjecture over the roles of TcdA and TcdB in CDI. Laboratory-derived TcdA and TcdB gene knock-out mutants, when tested in the gold standard hamster model, have given conflicting results. Using survival data, Lyras et al. (2009) proposed that TcdB was the key virulence factor. However, Kuehne et al. (2010, 2014) and Carter et al. (2015) have both demonstrated that either TcdA or TcdB can cause fulminant disease in the hamster infection model. Furthermore, in previous studies, we have shown that antibodies which neutralize specifically either TcdA or TcdB are both required to provide protection against CDI in the hamster when given systemically. In vitro assays demonstrated no neutralization of TcdA by anti-TcdB antibodies and vice versa (Roberts et al., 2012; Maynard-Smith et al., 2014). In this study we addressed if both antibodies are required for treatment of CDI in a hamster model when the therapy is given orally, and neutralizing antibodies are delivered directly to the site of 
infection. Our data showed that antibodies against TcdB alone did not significantly protect from CDI (Figure 5C). This finding suggests that $\mathrm{TcdA}$ is important in the pathogenesis of CDI, at least in the hamster model, when a highly toxigenic challenge strain is employed. Apart from their cytotoxic effects on the epithelium of the large intestine, TcdA and TcdB have been shown to be pro-inflammatory by eliciting cytokine release, with TcdA causing a greater pro-inflammatory reaction than TcdB (Yu et al., 2017). Therefore, the neutralization of both toxins might provide better protection of the gut epithelium. The strong inflammatory response induced by $\mathrm{TcdA}$ (and TcdB) triggers the release of inflammatory mediators from intestinal epithelial cells which leads to intestinal barrier dysfunction (Hansen et al., 2013; Yu et al., 2017). Thus, TcdA may play a pivotal role in the toxicity of $\mathrm{TcdB}$ and the severity of CDI by facilitating systemic entry of both toxins. Neutralization of both toxins at the site of their production might reduce gut damage, decreasing extra-intestinal transit of toxins and systemic effects of severe CDI. Kink and Williams (Kink and Williams, 1998), using orally delivered antibodies derived from eggs, showed that antibodies to both toxins were required to prevent mortality in the hamster model, supporting the results obtained in this study. However, Hutton et al. (2017) suggest that the oral administration of bovine hyperimmune colostrum, containing neutralizing antibodies to TcdB only, is effective in the treatment or prevention of CDI. There were several differences between the current study and the studies undertaken by Hutton and colleagues. For example, in the latter study, the mouse model of CDI was employed and run for 4 days post-challenge during which period mice were given hyper-immune bovine colostrum as the antibody source. A ribotype 027 C. difficile strain was used to challenge the mice. In our study, the hamster model was used and run for 16 days postinfection during which purified ovine antibodies against TcdA and TcdB were given for the first 4 days post-challenge only. The highly toxigenic ATCC 43255 strain of $C$. difficile produces high concentrations of TcdA and TcdB in vitro (Merrigan et al., 2010). The strain described by Merrigan et al. (2010) and the strain used in these studies was obtained from the same original source. With all these differences in methodology, it is unwise to compare the studies in detail. However, both studies support the hypothesis that toxin neutralizing antibodies can be delivered orally and prevent CDI in an animal model. It should also be noted that recently a variant strain of $C$. difficile, producing only $\operatorname{Tcd} A$ was isolated from humans (Marvaud et al., 2019), suggesting that antibodies that neutralize TcdA might be beneficial in CDI immune therapeutics.

\section{CONCLUSION}

OraCAb demonstrated potential to prevent CDI, induced by a highly toxigenic $C$. difficile strain, in the hamster model. A 3:1 ratio of anti-TxB4 IgG to anti-TxA4 IgG with a total IgG concentration of greater than $50 \mathrm{mg} / \mathrm{ml}$ IgG was required for protection of hamsters. In addition, using the in vitro gut model, it was proposed that OraCAb antibodies might be beneficial when used alongside vancomycin to prevent simulated recurrent CDI.
It is important to note that this in vitro model is limited to the microbiota of the gut, and cannot model some important factors in CDI, such has host immune response. It is likely that a treatment that prevents toxin (the disease causing agent) from acting on the gut mucosa in the first instance could allow (a) recovery of the microbiota to reduce further $C$. difficile growth (b) action of the immune system to reduce the burden of $C$. difficile. Clearly, clinical trials are required to answer such issues surrounding the capacity of orally administered antibodies to interrupt CDI/gut inflammation. With the preclinical development of OraCAb completed, the formulation needs to undergo a Phase $1 \mathrm{~b} / 2$ a clinical trial where will be tested for its safety and efficacy. The pre-clinical results presented here could be used as guidance for the design of antibody ratio and concentrations in OraCAb that will be used in clinical trials and subsequent product manufacture.

\section{DATA AVAILABILITY STATEMENT}

The raw data supporting the conclusions of this article will be made available by the authors, without undue reservation.

\section{ETHICS STATEMENT}

The studies involving human participants were reviewed and approved by the University of Leeds School of Medicine Research Ethics Committee (Ref: MREC15-070), Leeds, United Kingdom. The patients/participants provided their informed consent to participate in this study. The animal study was reviewed and approved by Ethical Review Process of Public Health England (PHE), Porton, Salisbury, United Kingdom and the Home Office, United Kingdom.

\section{AUTHOR CONTRIBUTIONS}

$\mathrm{AR}$ and $\mathrm{CS}$ conceived and designed the experiments performed at $\mathrm{PHE}$. $\mathrm{HH}, \mathrm{CC}, \mathrm{AB}$, and $\mathrm{MW}$ designed the in vitro gut model experiments. IM, DE, EC, and WS conducted the in vitro gut model and analyzed samples taken from the models. RD, JG, and OP developed the OraCAb formulation and developed and validated the toxin neutralization assay and BAPNA assay for activity of trypsin inhibitors. MS developed the purification protocol for the Bowman-Birk Inhibitor and helped with preparation of samples for the in vivo studies. AR also prepared the samples for use in these studies. AR, HH, CC, and RD wrote the manuscript. All authors discussed the results and commented on the manuscript.

\section{FUNDING}

This work was supported by a Biomedical Catalyst Award (Innovate UK), Project Number 102845 and SMARTCymru Project Reference Number 1086-ED-RDI 286. This research was also supported, in part, by UK Department of Health 
(AR, MS, and CS). The funders had no role in study design, data collection and interpretation, or the decision to submit the work for publication. The views expressed in this publication are those of the authors and not necessarily those of the UK National Health Service, UK Department of Health.

\section{ACKNOWLEDGMENTS}

We would like to thank the staff of the Biological Investigations Group at PHE for their excellent technical assistance in undertaking the in vivo experiments

\section{REFERENCES}

Babcock, G. J., Broering, T. J., Hernandez, H. J., Mandell, R. B., Donahue, K., Boatright, N., et al. (2006). Human monoclonal antibodies directed against toxins A and B prevent Clostridium difficile-induced mortality in hamsters. Infect. Immun. 74, 6339-6347. doi: 10.1128/IAI.00982-06

Baines, S. D., O'Connor, R., Saxton, K., Freeman, J., and Wilcox, M. H. (2008). Comparison of oritavancin versus vancomycin as treatments for clindamycininduced Clostridium difficile PCR ribotype 027 infection in a human gut model. J. Antimicrob. Chemother. 62, 1078-1085. doi: 10.1093/jac/dkn358

Borgstrom, B., Dahlqvist, A., Lundh, G., and Sjovall, J. (1957). Studies of intestinal digestion and absorption in the human. J. Clin. Invest. 36, 1521-1536. doi: 10.1172/JCI103549

Carter, G. P., Chakravorty, A., Pham Nguyen, T. A., Mileto, S., Schreiber, F., Li, L., et al. (2015). Defining the roles of TcdA and $\mathrm{TcdB}$ in localized gastrointestinal disease, systemic organ damage, and the host response during Clostridium difficile infections. MBio 6:e00551. doi: 10.1128/mBio.00551-15

Chaudhry, R., Sharma, N., Gupta, N., Kant, K., Bahadur, T., Shende, T. M., et al. (2017). Nagging presence of Clostridium difficile associated Diarrhoea in North India. J. Clin. Diagn. Res. 11, DC06-DC09. doi: 10.7860/JCDR/2017/29096. 10592

Chilton, C. H., Crowther, G. S., Baines, S. D., Todhunter, S. L., Freeman, J., Locher, H. H., et al. (2014a). In vitro activity of cadazolid against clinically relevant Clostridium difficile isolates and in an in vitro gut model of C. difficile infection. J. Antimicrob. Chemother. 69, 697-705. doi: 10.1093/jac/dkt411

Chilton, C. H., Crowther, G. S., Freeman, J., Todhunter, S. L., Nicholson, S., Longshaw, C. M., et al. (2014b). Successful treatment of simulated Clostridium difficile infection in a human gut model by fidaxomicin first line and after vancomycin or metronidazole failure. J. Antimicrob. Chemother. 69, 451-462. doi: $10.1093 / \mathrm{jac} / \mathrm{dkt} 347$

Chilton, C. H., Freeman, J., Crowther, G. S., Todhunter, S. L., and Wilcox, M. H. (2012). Effectiveness of a short (4 day) course of oritavancin in the treatment of simulated Clostridium difficile infection using a human gut model. J. Antimicrob. Chemother. 67, 2434-2437. doi: 10.1093/jac/dks243

Clemente, A., Sonnante, G., and Domoney, C. (2011). Bowman-Birk inhibitors from legumes and human gastrointestinal health: current status and perspectives. Curr. Protein Pept. Sci. 12, 358-373. doi: $10.2174 / 138920311796391133$

Cole, L. E., Li, L., Jetley, U., Zhang, J., Pacheco, K., Ma, F., et al. (2019). Deciphering the domain specificity of C. difficile toxin neutralizing antibodies. Vaccine 37, 3892-3901. doi: 10.1016/j.vaccine.2019.05.040

Crowe, J. S., Roberts, K. J., Carlton, T. M., Maggiore, L., Cubitt, M. F., Clare, S., et al. (2018). Preclinical development of a novel, orally-administered antitumour necrosis factor domain antibody for the treatment of inflammatory bowel disease. Sci. Rep. 8:4941. doi: 10.1038/s41598-018-23277-7

Crowther, G. S., Chilton, C. H., Todhunter, S. L., Nicholson, S., Freeman, J., and Wilcox, M. H. (2015). Recurrence of dual-strain Clostridium difficile infection in an in vitro human gut model. J. Antimicrob. Chemother. 70, 2316-2321. doi: $10.1093 / \mathrm{jac} / \mathrm{dkv} 108$

DeFilipp, Z., Bloom, P. P., Torres Soto, M., Mansour, M. K., Sater, M. R. A., Huntley, M. H., et al. (2019). Drug-resistant E. coli bacteremia transmitted described in this paper. We would also like to thank Miss. Kate Owen and Mrs. Sharie Shearman for their excellent technical assistance with the in vitro gut model and Ms. Joanna McGlashan for undertaking cellbased assays at PHE.

\section{SUPPLEMENTARY MATERIAL}

The Supplementary Material for this article can be found online at: https://www.frontiersin.org/articles/10.3389/fmicb. 2020.578903/full\#supplementary-material

by fecal microbiota transplant. N. Engl. J. Med. 381, 2043-2050. doi: 10.1056/ NEJMoa1910437

Freeman, J., Baines, S. D., Jabes, D., and Wilcox, M. H. (2005). Comparison of the efficacy of ramoplanin and vancomycin in both in vitro and in vivo models of clindamycin-induced Clostridium difficile infection. J. Antimicrob. Chemother. 56, 717-725. doi: 10.1093/jac/dki321

Freeman, J., O'Neill, F. J., and Wilcox, M. H. (2003). Effects of cefotaxime and desacetylcefotaxime upon Clostridium difficile proliferation and toxin production in a triple-stage chemostat model of the human gut. J. Antimicrob. Chemother. 52, 96-102. doi: 10.1093/jac/dkg267

Geratz, J. D., and Hurt, J. P. (1970). Regulation of pancreatic enzyme levels by trypsin inhibitors. Am. J. Physiol. 219, 705-711. doi: 10.1152/ajplegacy.1970. 219.3.705

Gerding, D. N., Johnson, S., Rupnik, M., and Aktories, K. (2014). Clostridium difficile binary toxin CDT: mechanism, epidemiology, and potential clinical importance. Gut Microbes 5, 15-27. doi: 10.4161/gmic.26854

Guh, A. Y., Mu, Y., Winston, L. G., Johnston, H., Olson, D., Farley, M. M., et al. (2020). Trends in U.S. burden of Clsotridioides difficile infection outcomes. N. Engl. J. Med. 382, 1320-1330. doi: 10.1056/NEJMoa1910215

Hansen, A., Alston, L., Tulk, S. E., Schenck, L. P., Grassie, M. E., Alhassan, B. F., et al. (2013). The P2Y6 receptor mediates Clostridium difficile toxin-induced CXCL8/IL-8 production and intestinal epithelial barrier dysfunction. PLoS One 8:e81491. doi: 10.1371/journal.pone.0081491

Harris, M. S., Hartman, D., Lemos, B. R., Erlich, E. C., Spence, S., Kennedy, S., et al. (2016). AVX-470, an orally delivered anti-tumour necrosis factor antibody for treatment of active ulcerative colitis: results of a first-in-human trial. J. Crohns Colitis 10, 631-640. doi: 10.1093/ecco-jcc/jjw036

Hutton, M. L., Cunningham, B. A., Mackin, K. E., Lyon, S. A., James, M. L., Rood, J. I., et al. (2017). Bovine antibodies targeting primary and recurrent Clostridium difficile disease are a potent antibiotic alternative. Sci. Rep. 7:3665. doi: 10.1038/s41598-017-03982-5

Jasion, V. S., and Burnett, B. P. (2015). Survival and digestibility of orallyadministered immunoglobulin preparations containing IgG through the gastrointestinal tract in humans. Nutr. J. 14:22. doi: 10.1186/s12937-015-0010-7

Jawa, R. S., and Mercer, D. W. (2012). Clostridium difficile-associated infection: a disease of varying severity. Am. J. Surg. 204, 836-842. doi: 10.1016/j.amjsurg. 2012.05.025

Kelly, C. P. (2012). Can we identify patients at high risk of recurrent Clostridium difficile infection? Clin. Microbiol. Infect. 18(Suppl. 6), 21-27. doi: 10.1111/ 1469-0691.12046

Kink, J. A., and Williams, J. A. (1998). Antibodies to recombinant Clostridium difficile toxins $\mathrm{A}$ and $\mathrm{B}$ are an effective treatment and prevent relapse of $\mathrm{C}$. difficile-associated disease in a hamster model of infection. Infect. Immun. 66, 2018-2025. doi: 10.1128/iai.66.5.2018-2025.1998

Kuehne, S. A., Cartman, S. T., Heap, J. T., Kelly, M. L., Cockayne, A., and Minton, N. P. (2010). The role of toxin A and toxin B in Clostridium difficile infection. Nature 467, 711-713. doi: 10.1038/nature09397

Kuehne, S. A., Collery, M. M., Kelly, M. L., Cartman, S. T., Cockayne, A., and Minton, N. P. (2014). Importance of toxin A, toxin B, and CDT in virulence of an epidemic Clostridium difficile strain. J. Infect. Dis. 209, 83-86. doi: 10.1093/ infdis/jit426 
Kyne, L., Warny, M., Qamar, A., and Kelly, C. P. (2001). Association between antibody response to toxin $\mathrm{A}$ and protection against recurrent Clostridium difficile diarrhoea. Lancet 357, 189-193. doi: 10.1016/S0140-6736(00)03592-3

Lee, Y., Lim, W. I., Bloom, C. I., Moore, S., Chung, E., and Marzella, N. (2017). Bezlotoxumab (Zinplava) for Clostridium Difficile Infection: the first monoclonal antibody approved to prevent the recurrence of a bacterial infection. P T 42, 735-738.

Lessa, F. C., Winston, L. G., McDonald, L. C., and Emerging Infections Program C. difficile Surveillance Team (2015). Burden of Clostridium difficile infection in the United States. N. Engl. J. Med. 372, 2369-2370. doi: 10.1056/NEJMc1505190

Liener, I. E., Goodale, R. L., Deshmukh, A., Satterberg, T. L., Ward, G., DiPietro, C. M., et al. (1986). Effect of trypsin inhibitor from soybeans (Bowman-Birk) on the secretory activity of the human pancreas. Gastroenterology 94, 419-427. doi: 10.1016/0016-5085(88)90431-3

Lyerly, D. M., Saum, K. E., MacDonald, D. K., and Wilkins, T. D. (1985). Effects of Clostridium difficile toxins given intragastrically to animals. Infect. Immun. 47, 349-352. doi: 10.1128/iai.47.2.349-352.1985

Lyras, D., O’Connor, J. R., Howarth, P. M., Sambol, S. P., Carter, G. P., Phumoonna, T., et al. (2009). Toxin B is essential for virulence of Clostridium difficile. Nature 458, 1176-1179. doi: 10.1038/nature07822

Macfarlane, G. T., Macfarlane, S., and Gibson, G. R. (1998). Validation of a three-stage compound continuous culture system for investigating the effect of retention time on the ecology and metabolism of bacteria in the human colon. Microb. Ecol. 35, 180-187. doi: 10.1007/s002489900072

Madoff, S. E., Urquiaga, M., Alonso, C. D., and Kelly, C. P. (2019). Prevention of recurrent Clostridioides difficile infection: a systematic review of randomized controlled trials. Anaerobe 61:102098. doi: 10.1016/j.anaerobe.2019.102098

Marvaud, J. C., Quevedo-Torres, S., Eckert, C., Janoir, C., and Barbut, F. (2019). Virulence of new variant strains of Clostridium difficile producing only toxin A or binary toxin in the hamster model. New Microbes New Infect. 32:100590. doi: 10.1016/j.nmni.2019.100590

Maynard-Smith, M., Ahern, H., McGlashan, J., Nugent, P., Ling, R., Denton, H., et al. (2014). Recombinant antigens based on toxins A and B of Clostridium difficile that evoke a potent toxin-neutralising immune response. Vaccine 32, 700-705. doi: 10.1016/j.vaccine.2013.11.099

McFarland, L. V., Surawicz, C. M., Rubin, M., Fekety, R., Elmer, G. W., and Greenberg, R. N. (1999). Recurrent Clostridium difficile disease: epidemiology and clinical characteristics. Infect. Control. Hosp. Epidemiol. 20, 43-50. doi: $10.1086 / 501553$

Merrigan, M., Venugopal, A., Mallozzi, M., Roxas, B., Viswanathan, V. K., Johnson, S., et al. (2010). Human hypervirulent Clostridium difficile strains exhibit increased sporulation as well as robust toxin production. J. Bacteriol. 192, 4904-4911. doi: 10.1128/JB.00445-10

Mullane, K. (2014). Fidaxomicin in Clostridium difficile infection: latest evidence and clinical guidance. Ther. Adv. Chronic Dis. 5, 69-84. doi: 10.1177/ 2040622313511285

Ofori, E., Ramai, D., Dhawan, M., Mustafa, F., Gasperino, J., and Reddy, M. (2018). Community-acquired Clostridium difficile: epidemiology, ribotype, risk factors, hospital and intensive care unit outcomes, and current and emerging therapies. J. Hosp. Infect. 99, 436-442. doi: 10.1016/j.jhin.2018.01.015

Pizarro-Guajardo, M., Diaz-Gonzalez, F., Alvarez-Lobos, M., and Paredes-Sabja, D. (2017). Characterization of chicken IgY specific to Clostridium difficile R20291 spores and the effect of oral administration in mouse models of initiation and recurrent disease. Front. Cell. Infect. Microbiol. 7:365. doi: 10.3389/fcimb.2017. 00365

Public Health England (2019). Annual Epidemiological Commentary: GramNegative Bacteraemia, MRSA Bacteraemia, MSSA Bacteraemia and C. Difficile
Infections, up to and Including Finacial Year April 2018 to March 2019. London: Public Health England.

Roberts, A., McGlashan, J., Al-Abdulla, I., Ling, R., Denton, H., Green, S., et al. (2012). Development and evaluation of an ovine antibody-based platform for treatment of Clostridium difficile infection. Infect. Immun. 80, 875-882. doi: 10.1128/IAI.05684- 11

Saxena, I., and Tayyab, S. (1997). Protein proteinase inhibitors from avian egg whites. Cell. Mol. Life Sci. 53, 13-23. doi: 10.1007/pl00000575

Shearman, D. J., Parkin, D. M., and McClelland, D. B. (1972). The demonstration and function of antibodies in the gastrointestinal tract. Gut 13, 483-499. doi: 10.1136/gut.13.6.483

Sponseller, J. K., Steele, J. A., Schmidt, D. J., Kim, H. B., Beamer, G., Sun, X., et al. (2015). Hyperimmune bovine colostrum as a novel therapy to combat Clostridium difficile infection. J. Infect. Dis. 211, 1334-1341. doi: 10.1093/infdis/ jiu605

Steele, J., Mukherjee, J., Parry, N., and Tzipori, S. (2013). Antibody against TcdB, but not TcdA, prevents development of gastrointestinal and systemic Clostridium difficile disease. J. Infect. Dis. 207, 323-330. doi: 10.1093/infdis/ jis669

Watson, D. L. (1992). Biological half-life of ovine antibody in neonatal lambs and adult sheep following passive immunization. Vet. Immunol. Immunopathol. 30, 221-232. doi: 10.1016/0165-2427(92)90140-1

Wiegand, P. N., Nathwani, D., Wilcox, M. H., Stephens, J., Shelbaya, A., and Haider, S. (2012). Clinical and economic burden of Clostridium difficile infection in Europe: a systematic review of healthcare-facility-acquired infection. J. Hosp. Infect. 81, 1-14. doi: 10.1016/j.jhin.2012.02.004

Wilcox, M. H., Gerding, D. N., Poxton, I. R., Kelly, C., Nathan, R., Birch, T., et al. (2017). Bezlotoxumab for prevention of recurrent Clostridium difficile infection. N. Engl. J. Med. 376, 305-317. doi: 10.1056/NEJMoa1602615

Worning, H., and Müllertz, S. (1966). pH and pancreatic enzymes in the human duodenum during digestion of a standard meal. Scand. J. Gastroenterol. 1, 268-283. doi: 10.1080/00365521.1966.11800642

Yu, H., Chen, K., Sun, Y., Carter, M., Garey, K. W., Savidge, T. C., et al. (2017). Cytokines are markers of the Clostridium difficile-induced inflammatory response and predict disease severity. Clin. Vaccine Immunol. 24, e00037-17. doi: 10.1128/CVI.00037-17

Zhang, Z., Chen, X., Hernandez, L. D., Lipari, P., Flattery, A., Chen, S. C., et al. (2015). Toxin-mediated paracellular transport of antitoxin antibodies facilitates protection against Clostridium difficile infection. Infect. Immun. 83, 405-416. doi: 10.1128/IAI.02550-14 doi: 10.1128/iai.02550-14

Conflict of Interest: RD, JG, and OP were employed by the company MicroPharm Ltd. AR and CS hold patent WO 2011/067616, "Therapies for preventing or suppressing Clostridium difficile infection.” AR, CS, and MS hold patent WO 2012/046061, "Clostridium difficile antigens."

The remaining authors declare that the research was conducted in the absence of any commercial or financial relationships that could be construed as a potential conflict of interest.

Copyright (c) 2020 Roberts, Harris, Smith, Giles, Polak, Buckley, Clark, Ewin, Moura, Spitall, Shone, Wilcox, Chilton and Donev. This is an open-access article distributed under the terms of the Creative Commons Attribution License (CC BY). The use, distribution or reproduction in other forums is permitted, provided the original author(s) and the copyright owner(s) are credited and that the original publication in this journal is cited, in accordance with accepted academic practice. No use, distribution or reproduction is permitted which does not comply with these terms. 